\title{
Halide Segregation in Mixed Halide Perovskites: Visualization and Mechanisms
}

\author{
Liuwen Tian ${ }^{1,2}$, Jingjing Xue ${ }^{1,3}$ and Rui Wang ${ }^{2, *(D)}$ \\ 1 School of Materials Science and Engineering, Zhejiang University, Hangzhou 310027, China; \\ tianliuwen@westlake.edu.cn (L.T.); jjxue@zju.edu.cn (J.X.) \\ 2 School of Engineering, Westlake University, 18 Shilongshan Road, Hangzhou 310024, China \\ 3 Shanxi-Zheda Institute of Advanced Materials and Chemical Engineering, Zhejiang University, \\ Hangzhou 310027, China \\ * Correspondence: wangrui@westlake.edu.cn
}

Citation: Tian, L.; Xue, J.; Wang, R. Halide Segregation in Mixed Halide Perovskites: Visualization and Mechanisms. Electronics 2022, 11, 700. https://doi.org/10.3390/ electronics 11050700

Academic Editor: Elias Stathatos

Received: 16 February 2022 Accepted: 22 February 2022 Published: 24 February 2022

Publisher's Note: MDPI stays neutral with regard to jurisdictional claims in published maps and institutional affiliations.

Copyright: (C) 2022 by the authors. Licensee MDPI, Basel, Switzerland. This article is an open access article distributed under the terms and conditions of the Creative Commons Attribution (CC BY) license (https:// creativecommons.org/licenses/by/ $4.0 /)$.

\begin{abstract}
Photoinduced halide segregation in mixed halide perovskites is an intriguing phenomenon and simultaneously a stability issue. In-depth probing this effect and unveiling the underpinning mechanisms are of great interest and significance. This article reviews the progress in visualized investigation of halide segregation, especially light-induced, by means of spatially-resolved imaging techniques. Furthermore, the current understanding of photoinduced phase separation based on several possible mechanisms is summarized and discussed. Finally, the remained open questions and future outlook in this field are outlined.
\end{abstract}

Keywords: perovskite; halide segregation; direct observation; imaging techniques; mechanism

\section{Introduction}

Metal halide perovskites (MHPs), as a class of unique semiconductor materials, have sparked unprecedentedly intense research activities globally over the past decade. This is mainly due to their splendid optoelectronic properties and ease of processing, enabling efficiently fabricating high-performance photovoltaic devices with low-cost. Tremendous advances of perovskite solar cells (PSCs) have been achieved in terms of power conversion efficiencies (PCEs), large-area fabrication and device stability since their emergence in 2009, especially after 2012, making PSCs become significant candidates for next-generation photovoltaic technologies [1-12]. In addition, MHPs are attractive and promising for other applications beyond photovoltaics, including light-emitting diodes (LEDs) [13-15], photocatalysis [16,17], photodetectors [18,19], lasers [20,21], transistors [22,23], thermoelectrics [24], and even nonlinear optics [25], spintronics [26] and so forth, demonstrating MHPs are indeed a type of versatile and fascinating materials.

In general, MHPs feature a chemical formula of $\mathrm{ABX}_{3}$, where $\mathrm{A}$ refers to methylammonium, formamidinium or cesium cation $\left(\mathrm{MA}^{+}=\mathrm{CH}_{3} \mathrm{NH}_{3}{ }^{+}, \mathrm{FA}^{+}=\mathrm{HC}\left(\mathrm{NH}_{2}\right)_{2}{ }^{+}, \mathrm{or} \mathrm{Cs}^{+}\right)$, $\mathrm{B}$ is lead or tin cation $\left(\mathrm{Pb}^{2+}\right.$ or $\left.\mathrm{Sn}^{2+}\right)$, $\mathrm{X}$ denotes halide anion $\left(\mathrm{I}^{-}, \mathrm{Br}^{-}\right.$or $\left.\mathrm{Cl}^{-}\right)$. The $\mathrm{BX}_{6}$ octahedra with corner-sharing form a three-dimensional framework, and A-site cations are centrally located in the cuboctahedral cavities $[27,28]$. The unique crystal structure, moderate $\mathrm{Pb}$-I bonding energy, and weak electrostatic interaction together with hydrogen bonding between the organic A cations and halogen anions coherently dictate MHPs featuring soft nature [27]. This character makes MHPs be of easy preparation and processing, but also face inherent instability issues, such as ion migration, halide segregation, phase transition and degradation [29-33].

As is known, bandgap tunability is one of the most attractive characteristics for MHPs (from about 1.2 to $3.0 \mathrm{eV}$ ) [34,35], which can be readily achieved through compositional engineering, offering immense opportunities to fabricate perovskite based multijunction photovoltaics and multicoloured LEDs by meticulously regulating the energy bandgap to 
the target values of the perovskites. However, an apparitional phenomenon existing in MHPs, called halide segregation, makes such anticipations far from favorableness [31]. The halide segregation occurs when MHPs exposed to photoirradiation or subjected to charge carrier injection. As a result, coessential aggregation of iodide (I) and bromide (Br) occurs, leading to the formation of I-rich and Br-rich regions. The I-rich domains feature narrower energy bandgaps, acting as radiative recombination centers due to carriers funneling from adjacent Br-rich regions, thus giving rise to redshifted photoluminescence (PL) emission. What is even more peculiar is that when the perovskite films are kept in the dark on a timescale of from minutes to hours, remixing processes take place and the corresponding films could recover to their original states. Since photoinduced halide segregation in MHPs was first reported by Hoke et al. in 2015 [31], numerous studies have been devoting to this subject, striving to uncover the microscopic mechanism and reveal what is the driving force behind the phenomenon or seek effective strategies to suppress it.

The fast-growing publications related to halide segregation in MHPs, as shown in Figure 1, indicate that halide segregation has been arousing tremendous attention in the perovskite community. However, thus far, a consensus has yet to be reached on the full underlying mechanisms for light-induced halide segregation. The construction of an ultimate theory or mechanism necessitates the guidance and support from direct observation on halide segregation using imaging techniques with high spatial resolution. Over the past few years, a number of studies have focused on this research direction. Some microscopy techniques, including PL mapping, cathodoluminescence (CL) imaging, transmission electron microscopy (TEM), energy-dispersive X-ray spectroscopy (EDS), atomic force microscopy (AFM), have been utilized to visualize halide demixing process and image spatial distribution and evolution of I-rich and Br-rich domains in MHPs. The obtained results provide direct and powerful evidences that help the perovskite community to get in-depth insight into halide segregation and ultimately uncover the underlying mechanism.

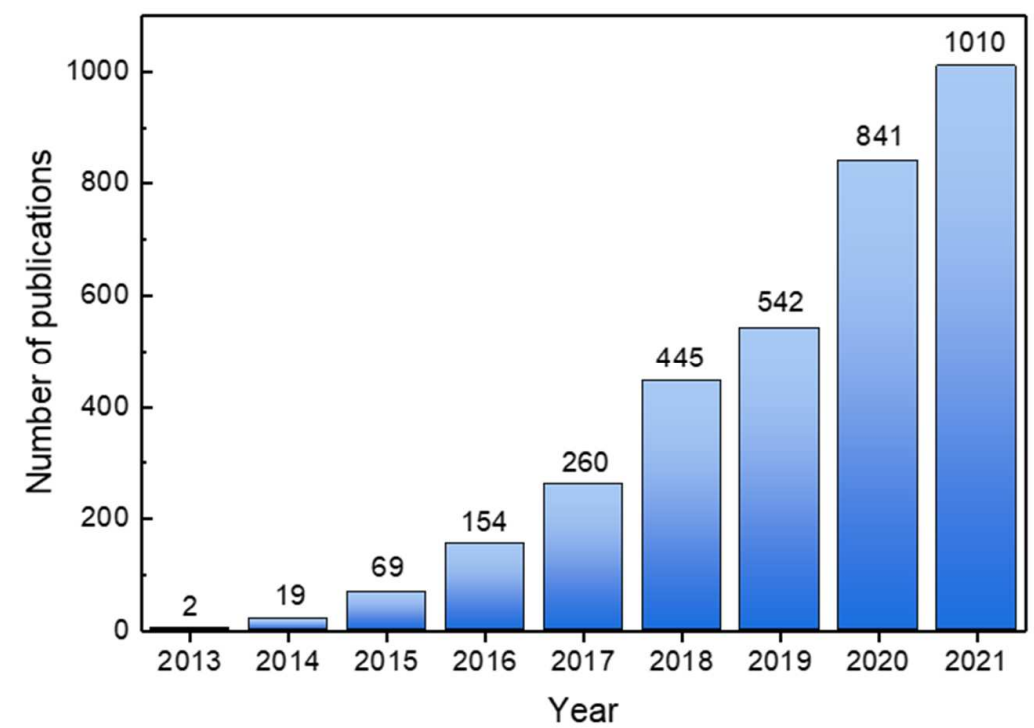

Figure 1. The number of publications in each year regarding halide segregation in metal halide perovskites. Note that the data might be somewhat overestimated.

Several review papers focusing on halide segregation in MHPs have been published, most of which take great attention on reviewing and discussing the origin, impact and suppression of halide segregation [36-45]. While we notice that the research progress on imaging halide segregation has yet to be comprehensively reviewed, which is of particular importance to deep understand this effect. Herein, we present this review article to systematically summarize and discuss the findings and advances in visualized investigation on phase separation, especially photoinduced, in terms of different characterization methods. Additionally, we review and discuss the currently prevailing possible mechanisms or theo- 
retical models accounting for this phenomenon. Finally, the remained open questions and future outlook in this field are outlined.

\section{Visualization of Halide Segregation}

Several years ago, Hoke and coworkers first noticed that the PL spectra of $M A P b\left(B r_{x} I_{1-x}\right)_{3}$ MHPs could undergo a red-shift when the perovskite films were exposed to illumination in less than one minute, which, interestingly, would recover to their original states when the films were kept in the dark for a few minutes [31]. Inspired by this behavior, they discovered the light-induced halide segregation phenomenon in MHPs. Since then, significant research activities have been focusing on this subject. During this process, recording the variation of steady-state PL spectra, ultraviolet-visible (UV-vis) absorption spectra, and transient absorption spectra are simple and effective ways to monitor and evaluate the phase segregation. While, in-depth understanding of this phenomenon necessitates locally multimodal microscale imaging of halide segregation. In this section, the advances in visualized investigation of halide demixing in many cases based on different characterization methods are systematically reviewed and discussed.

\subsection{Photoluminescence Mapping}

Photoluminescence (PL) measurements represent the most extensively adopted method to characterize the charge carrier recombination behavior of perovskites. A photoexcited electron from the excited state returns to ground state through radiative pathway, leading to a photon emitting. As electron-hole pairs generally thermalize to the band edges before recombination, the wavelength of emission peak directly correlates with the bandgap of the perovskite material. Thus, detecting and analyzing the emitted photons provides insight into the photoelectric quality and band gap of the perovskite in terms of emission intensity and wavelength [46]. Furthermore, spatially resolved PL mapping enabled by PL microscopy technique can give significant information of spatial variations in the photogenerated carrier recombination dynamics, which can be used to determine the film quality, heterogeneity, trap state distribution, ion migration, local phase transformation and so forth [29,47-55]. PL microscopy generally includes confocal and widefield types. The former can deliver PL images with much higher spatial resolution than the latter, owing to adopting a point light source and single point detector. For local visualization of halide segregation in MHPs under external stimuli, PL mapping, featuring noninvasive, nondestructive characteristics, desirable operational flexibility, and relative ease of access, is naturally becoming the most widely used characterization method.

Some previous works based on confocal PL spectroscopy or PL microscopy characterization suggested that halide segregation is preferable to occur at the grain boundaries for polycrystalline films and the edges for monocrystalline microplates. Tang et al. [56] compared the PL emission behavior evolution over time of the grain boundaries and grain interior under illumination by using shear-force scanning probe microscopy combined with confocal optical spectroscopy. They observed distinct PL emission variation with a new emission peak appearing at the longer wavelength when the laser spot was focused on grain boundaries just after $100 \mathrm{~s}$ of illumination. When the laser spot was placed on the center of grain, the PL emission was invariable over a period of $600 \mathrm{~s}$ (see Figure 2A). This suggests that light-induced phase segregation is initially preferable to occur at the grain boundaries. This result was confirmed by another group using the same characterization method [57]. Additionally, in this work, the researchers found that photoinduced phase segregation selectively appears at grain boundaries for MA-perovskite films with $35 \% \mathrm{Br}$ concentration, while for $65 \% \mathrm{Br}$ samples, halide demixing occurs over the whole films, including grain interior (Figure 2B). Furthermore, the results of PL mapping performed on $\mathrm{CsPbIBr}_{2}$ polycrystalline films upon light exposure reported by Yin et al. [58] also support this conclusion. Red shifted PL emission can be clearly observed at the grain boundaries after light soaking for $240 \mathrm{~s}$, indicating the formation of I-rich phase in these places. While, more interestingly, they noticed that sporadic I-rich regions assigning to some entire grains 
promptly appeared throughout the perovskite films under illumination, forming emission islands with longer wavelength.

A

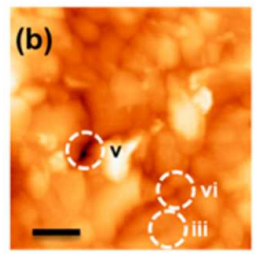

B
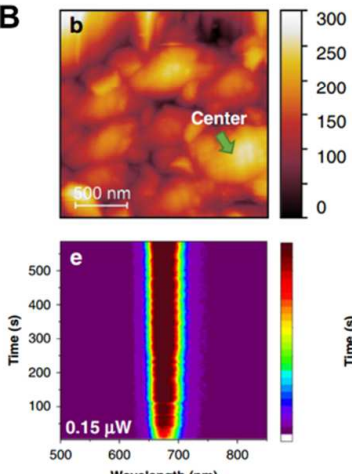
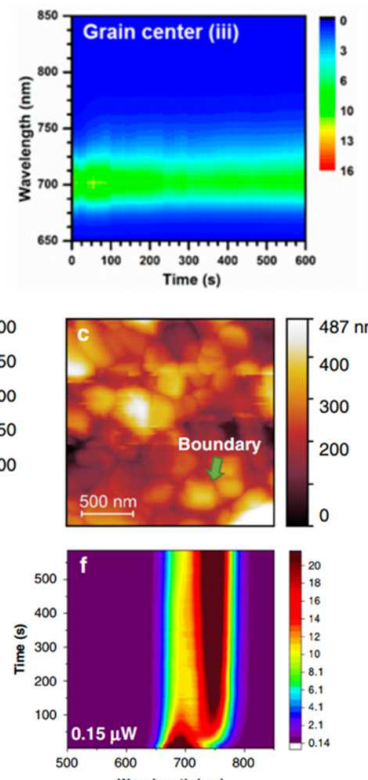
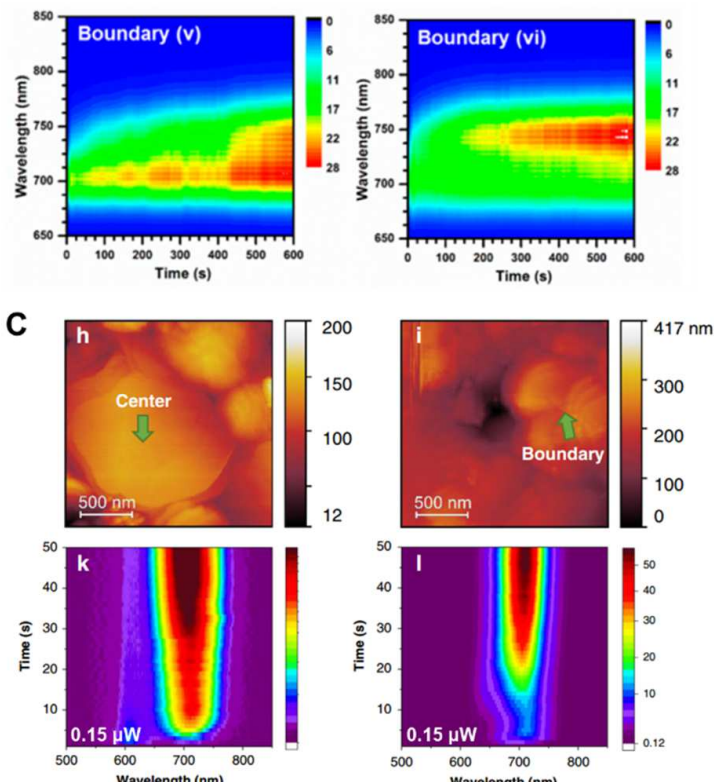

Figure 2. Local PL spectra changes over time. (A) Shear-force topographic image of a $\mathrm{MAPb}\left(\mathrm{I}_{0.7} \mathrm{Br}_{0.3}\right)_{3}$ perovskite film and corresponding PL spectra evolution over time at different locations as marked. Reproduced with permission [56]. Copyright 2018, American Chemical Society. (B) Shear-force topographic images of a $\mathrm{MAPb}\left(\mathrm{I}_{0.65} \mathrm{Br}_{0.35}\right)_{3}$ perovskite film and $\mathrm{PL}$ spectra evolution as a function of time at grain interior and grain boundary. (C) Shear-force topographic images of a $\mathrm{MAPb}\left(\mathrm{I}_{0.35} \mathrm{Br}_{0.65}\right)_{3}$ perovskite film and PL spectra evolution with respect to time at grain center and grain boundary. Reproduced with permission [57]. Copyright 2020, Nature Publishing Group.

In another work, Tiede and coworkers [59] demonstrated that the PL dark regions of the origin film were more susceptible to photoinduced halide segregation to form I-rich phase under illumination, suggesting that halide segregation rate is positively correlated with defect density. Moreover, they also observed that halide demixing domains expanded far beyond the illuminated area, which is indicative of light-induced halide ion migration extends beyond the illuminated region. Hence, this work provides visualized evidence of the strong correlation between halide segregation and defects or trap states, which has also been widely demonstrated by other works, despite lack of such direct and spatial resolved observation [60-65]. Although the exact mechanism of how defects or trap states accelerate phase segregation have yet to be well understood, halide vacancies mediated halide anion migration and trap states induced photogenerated carriers trapping are considered to play crucial roles in phase separation $[38,60,61,63,66,67]$. In this context, it is easy to understand why halide demixing generally occurs at the grain boundaries and some entire grains at first, as observed by PL mapping. Because grain boundaries are commonly high defective regions $[54,68,69]$. Besides, the heterogeneity of halide perovskite thin films has been extensively demonstrated [47-49,54,68,70]. The electronic quality could vary from grain to grain, and photoexcited carriers in some grains can dominantly recombine through non-radiative channels, leading to the formation of PL dark regions [48,49]. Furthermore, there are some other works that utilize PL mapping to investigate halide segregation of polycrystalline MHP films. While due to relatively long illumination time and/or low spatial resolution, they are unable to clearly deliver the information about local variation or distribution of phase segregation at the nanoscale or microscale [71-74]. Thus, we would like to skip over them.

As discussed above, defects in perovskite films have great impact on halide segregation. Thus, it is natural to wonder whether halide segregation is an intrinsic material property or 
not for MHPs [75]. Besides, in polycrystalline perovskite films, there exists many factors, such as morphology, grain size, grain boundaries, halide defects, and surface states that can influence phase segregation, making it unable to decouple the role of each factor $[75,76]$. Thus, investigation of halide segregation in monocrystalline MHPs is essential. Bischak et al. [75] prepared monocrystalline $\mathrm{MAPb}\left(\mathrm{Br}_{\mathrm{x}} \mathrm{I}_{1-\mathrm{x}}\right)_{3}$ nanoplates and used confocal PL microscopy to visualize the halide demixing behavior over time in these single crystals under illumination. They found that phase segregation phenomenon also readily occurred in these as-prepared monocrystalline nanoplates under light soaking just for $10 \mathrm{~s}$. This might indicate that photoinduced phase separation is an intrinsic property of MHPs, at least for MA cation based MHPs. While, by partially replacing MA cation with Cs cation, significantly suppressed halide segregation are observed, as seen in Figure 3. The authors attribute this phenomenon to the reduced electron-phonon coupling strength upon addition of Cs cation. It is noted that the addition of Cs cation into mixed halide perovskites has been widely proven to be one of the most effective strategies to mitigate phase segregation, even though a consensus in the community has yet to be reached on the full underlying mechanism how Cs cation affect halide segregation dynamics, that is, the reduction in electron-phonon coupling strength is not the only explanation in the literature [77-83].

A
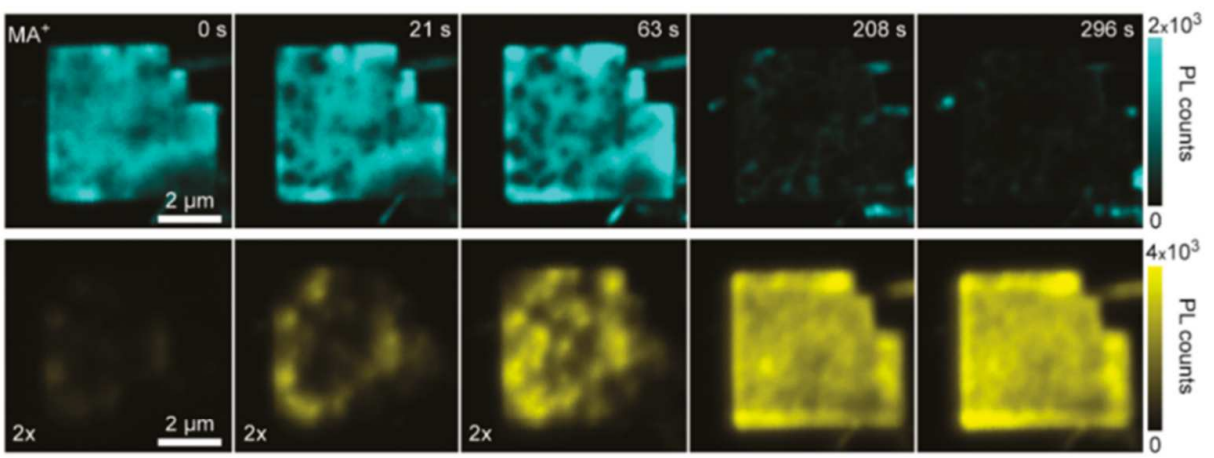

B

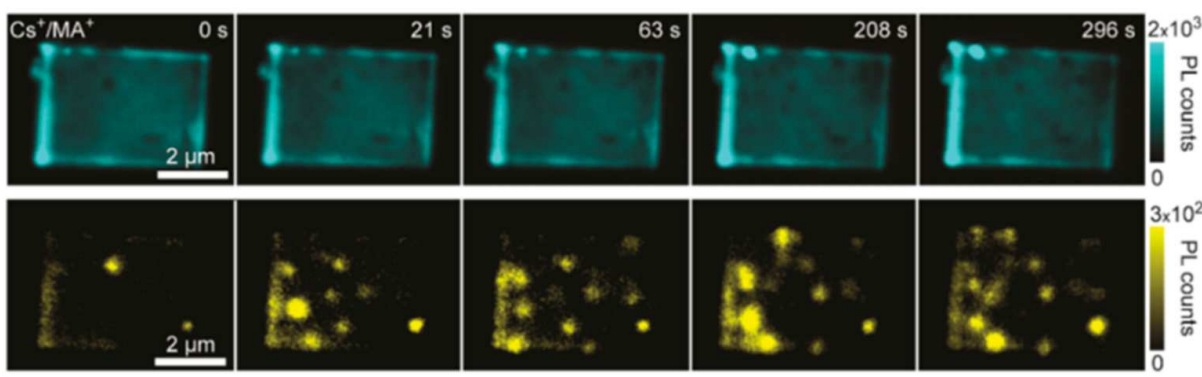

Figure 3. Confocal PL maps of (A) $\mathrm{MAPb}\left(\mathrm{I}_{0.14} \mathrm{Br}_{0.86}\right)_{3}$ and (B) $\mathrm{Cs}_{0.39} \mathrm{MA}_{0.61} \mathrm{~Pb}\left(\mathrm{I}_{0.15} \mathrm{Br}_{0.85}\right)_{3}$ monocrystalline nanoplates. The blue channel shows the PL emission of the original mixed phase (500-560 nm), and the yellow channel corresponds to the PL emission of segregated I-rich domains (650-750 nm). Reproduced with permission [75]. Copyright 2018, American Chemical Society.

Visualizing halide segregation in MHP single crystals using PL imaging technique was also conducted by Mao and colleagues [76]. I-rich clusters are observed throughout the entire $\mathrm{MAPb}\left(\mathrm{Br}_{\mathrm{x}} \mathrm{I}_{1-\mathrm{x}}\right)_{3}$ monocrystalline microplate, which is in agreement with the result reported by Bischak et al. [75], highlighting grain boundaries are not essential for phase segregation. However, there also exists some incompatible results between the two studies. First, in the work reported by Mao et al. [76], microplate edges exhibit much notable red-shifted PL emission than the interior after light soaking, which may mean that the formation of I-rich phase at the edges is much more favorable than in the interior. While in the work reported by Bischak and coworkers [75], it is not this case. In turn, it is the mixed phase shows more notable PL emission at the edges of the microplate, which is considered to likely stem from either photonic waveguiding effect [84] or a lower concentration of MA vacancies at the edges [85], and there is no such phenomenon of red- 
shifted PL emission. Second, what is even more contradictory is that the two reports hold evidently different standpoints regarding whether the locations of segregated I-rich regions are fixed or changed when the light on/off induced halide demixing/remixing cycles are performed. Bischak et al. [75] observed that I-rich clusters would not repeatedly appear in the same locations based on CL imaging (see Figure 4A), while Mao and colleagues [76] found that I-rich domains constantly formed in fixed places by using PL mapping, as shown in Figure 4B. The reason accounting for this contradiction is remained unclear. Besides, Mao and coworkers [76] also found that the segregated I-rich clusters feature two different emission wavelength regions, indicating the compositions of the I-rich phase are far from homogeneous.

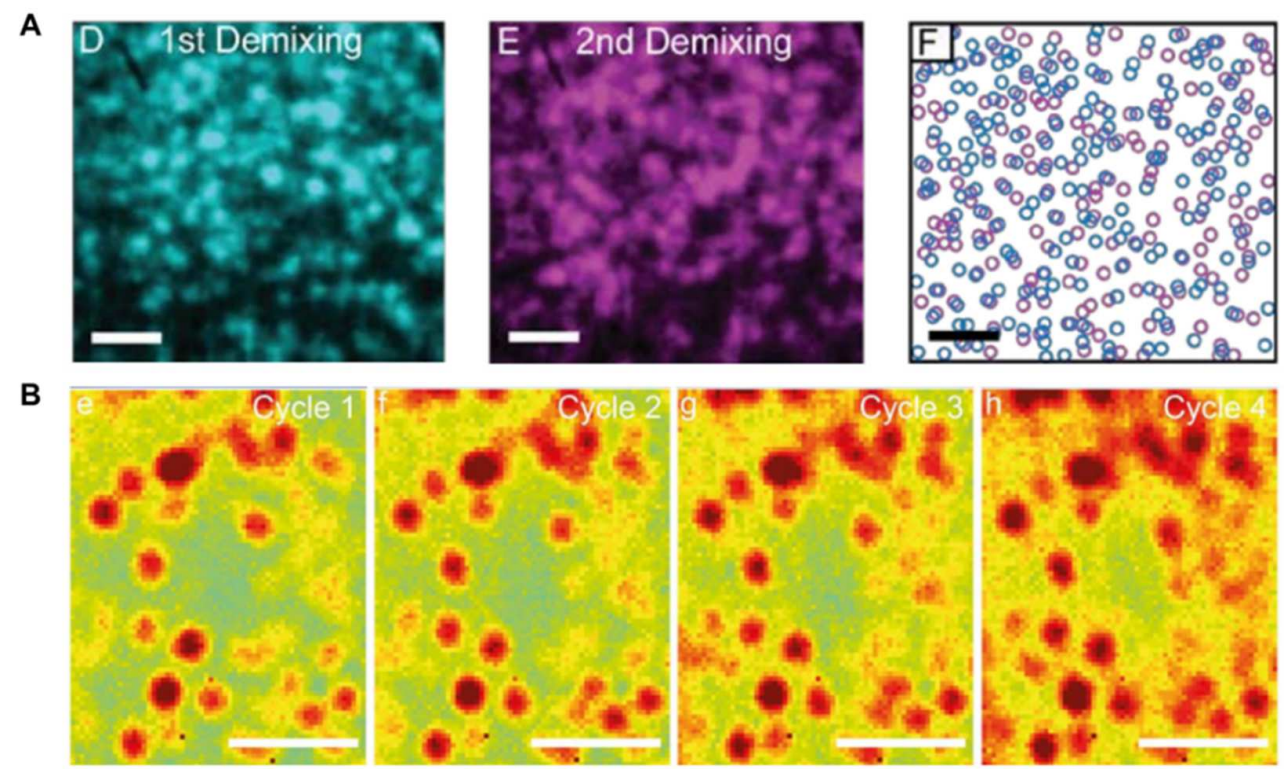

Figure 4. (A) CL images of a specific region of a $\mathrm{MAPb}\left(\mathrm{I}_{0.14} \mathrm{Br}_{0.86}\right)_{3}$ monocrystalline nanoplate from the first and second light-induced segregation steps, and an overlay of the local emission maxima in the first (blue) and second (pink) segregation steps, respectively. Reproduced with permission [75]. Copyright 2018, American Chemical Society. (B) PL maps of a target region of a $\mathrm{MAPb}\left(\mathrm{Br}_{\mathrm{x}} \mathrm{I}_{1-\mathrm{x}}\right)_{3}$ single crystal in four light on/off cycles. Reproduced with permission [76]. Copyright 2019, John Wiley \& Sons. The scale bars are $1 \mu \mathrm{m}$ for the three images in (A), and $2 \mu \mathrm{m}$ for the images in (B).

One year later, Mao and Chen et al. [86] reported their further investigation and understanding on the phase segregation dynamics in MHP single crystals by comparing one-photon and two-photon absorption based PL mapping. They ascertained that surface and edge states significantly impact photoinduced phase segregation. In the case of onephoton excitation, charge carriers are principally generated from the vicinity of perovskite surface with a minor contribution from the bulk, while two-photon excitation can penetrate the perovskite crystal, allowing the examination of bulk effects [86,87]. Similar to their previous work, I-rich phase related PL emission exhibits an obviously enhanced intensity at the edges of the perovskite monocrystalline microplate. Furthermore, under one-photon excitation, PL mapping shows that the I-rich PL emission is localized at the edges and almost unchanged over time. While, according to the time-resolved PL mapping under consecutive two-photon excitation, they observe that I-rich phase correlated PL emission firstly appears at the crystal edges, especially the corners where two edges are joining, and then gradually diffuse into the interior with the decrease of PL intensity corresponding to the parent phase, suggesting a direct I-Br substitution during this process. To interpret the halide segregation dynamics in MHPs single crystals observed in this study, the authors proposed a mechanism that upon light excitation, photogenerated charge carriers undergo diffusion, recombination, and surface trapping processes and the survived free carriers accumulate at the edges, which serve as a dam of the diffused carriers. The cumulation of 
charge carriers may result in strong electron-phonon coupling or higher potential energy in the crystal edges, thus triggering halide ions migration and ultimately phase segregation. In this context, the distinctions observed in one-photon and two-photon PL maps over time are considered to be derived from the different locations that charge carriers generated and the impact of surface defects on carrier recombination under one-photon and two-photon excitation situations.

\subsection{Cathodoluminescence Imaging}

Similar to PL mapping, cathodoluminescence (CL) imaging technique is also an important way to probe local halide segregation at the nanoscale. CL microscopy is generally equipped into scanning electron microscopy (SEM) system, imaging the luminescence of a material induced by electron beam. CL is quite sensitive to the optoelectronic properties of the materials and can achieve high spatial resolution with about $20 \mathrm{~nm}$, compared with a low spatial resolution of around $300 \mathrm{~nm}$ given by PL mapping at typical excitation/emission wavelengths due to diffraction limitation [88-90]. Simultaneously detecting secondary electrons and fluorescence emission enables to directly correlate microstructure with local carrier radiative recombination characteristics of the perovskites, thus shedding light on the spatial distributions of segregated I-rich phase with nanoscale resolution. While, of particular note is that care must be taken to avoid beam-induced damage during measurement, especially for hybrid perovskites with soft nature. Therefore, significantly reducing acceleration voltage and the probing current, even coupled with low-temperature cooling are required $[88,89]$.

Bischak and coworkers [77] first took advantage of the CL imaging method to visualize the spatial distribution of photoinduced I-rich clusters in $\mathrm{MAPb}\left(\mathrm{I}_{0.1} \mathrm{Br}_{0.9}\right)_{3}$ film after light soaking, which are demonstrated to primarily locate at the grain boundaries in steady state, as displayed in Figure 5B. Figure 5A is the corresponding SEM image, showing the morphology of the perovskite film. It is in this work that the authors proposed the famous polaron model to interpret the origin of photoinduced halide segregation based on experimental observations and multiscale modeling, which will be further discussed in the next section. They pointed out that reducing defect concentrations to restrict vacancy-mediated halide migration or weakening electron-phonon coupling can significantly suppress lightinduced phase separation, which are in good agreement with broadly experimental results. Subsequently, CL imaging focusing on all-inorganic $\mathrm{CsPbIBr}_{2}$ films to study local charge carrier recombination and phase segregation under illumination and electron beam irradiation was reported [91]. The researchers also observed that halide demixing preferred to occur at the grain boundaries and some grains, as shown in Figure 5C,D. They deem that phase segregation gives rise to massive mobile ions migrating along grain boundaries as ion movement highways and piling up at device interfaces, thus causing pronounced current density-voltage hysteresis of the corresponding photovoltaic cells. Duong et al. [71] also employed CL microscopy to imaging halide demixing and remixing processes of the as-prepared $\mathrm{Rb}_{0.05}\left(\mathrm{Cs}_{0.1} \mathrm{MA}_{0.15} \mathrm{FA}_{0.75}\right)_{0.95} \mathrm{PbI}_{2} \mathrm{Br}$ quadruple-cation perovskite film after light soaking under one-sun illumination and after being stored in the dark. According to the obtained CL images, significant photoinduced phase segregation occurred in many entire grains throughout the perovskite film after illumination for $12 \mathrm{~h}$, which could not completely recover to its original state even after being placed in the dark for $12 \mathrm{~h}$. On the basis of their previous work as introduced above [77], Bischak and colleagues [75] performed CL imaging, as a complementary tool for PL microscopy, to examine photoinduced phase separation in monocrystalline $\mathrm{MAPb}\left(\mathrm{Br}_{\mathrm{x}} \mathrm{I}_{1-\mathrm{x}}\right)_{3}$ nanoplate. They observed that redshifted CL corresponding to I-rich clusters quickly appeared and propagated throughout the perovskite nanoplate within tens of seconds under illumination at $405 \mathrm{~nm}$ with a light intensity of $100 \mathrm{~mW} \mathrm{~cm}^{-2}$, which is consistent with the PL mapping results. Moreover, by comparing the CL images of an identical area, researchers noticed that I-rich domains did not repeatedly appear in the same locations when the halide demixing/remixing reversible cycles were performed. However, such a viewpoint is under debate since an opposite 
experimental observation was reported by another group using PL mapping [76], which has been discussed above.

\subsection{Transmission Electron Microscopy}

Transmission electron microscopy (TEM) is also a powerful and widely used technique to probe the microstructures of materials by detecting the transmitted electrons through the specimen, enabling to achieve a resolution down to sub-angstrom [92]. Thus, the thickness of sample is generally controlled to be in the range of 5 to $100 \mathrm{~nm}$ [93], which depends on the elemental composition of the observed material and the acceleration voltage, to guarantee enough electron transparency. Funk et al. [94] have monitored halide segregation behavior in $\mathrm{CsPb}\left(\mathrm{Br}_{0.8} \mathrm{I}_{0.2}\right)_{3}$ perovskite under electron beam irradiation using in situ TEM. The authors hold the viewpoint that light and electron irradiation are equivalent in the aspect of inducing phase separation in various MHPs. They directly prepared the $\mathrm{CsPb}\left(\mathrm{Br}_{\mathrm{x}} \mathrm{I}_{1-\mathrm{x}}\right)_{3}$ samples on a carbon-coated TEM grid by spin-coating. After five minutes of electron beam irradiation, the abundance maps derived from the HRTEM images showed that the phase in the center of the nanoparticle was converted into $\mathrm{CsPbBr}_{3}$, while the phase close to the edges was in agreement with $\mathrm{CsPb}\left(\mathrm{Br}_{0.6} \mathrm{I}_{0.4}\right)_{3}$, indicating a $\mathrm{Br} / \mathrm{I}$ substitution process upon electron beam exposure, consistent with previous works using PL and CL imaging techniques as discussed above $[76,77,86]$.

\subsection{Energy-Dispersive X-ray Spectroscopy}

Energy-dispersive X-ray spectroscopy (EDS or EDX) is a basic and widely used technique to probe elemental composition and distribution, which is a typical accessory equipment integrated into the SEM and TEM systems. When the electron beam is irradiated onto the specimen, detecting and analyzing the characteristic $\mathrm{X}$-rays generated from the atoms using energy dispersive detector allows performing qualitative and quantitative elemental analysis [92]. The constant advance of SEM technique enables a spatial resolution down to one nanometer. While for general EDS, the resolutions are still restricted to about several hundred nanometers or beyond one micrometer for bulk specimens, which depend on material characteristics, sample thickness, acceleration voltage, and so forth. This restriction is due to the scattering of electrons inside the sample, leading to a large tear-drop shaped excitation volume below the surface where the characteristic X-rays are generated [95]. By contrast, the spatial resolution of several nanometers for EDS in TEM is realizable due to the sample size is generally in the order of a few tens to hundred nanometers in thickness. As such, it is impracticable to get insight into the local halide segregation at the nanoscale using SEM-EDS, but in principle, feasible by means of TEM-EDS system.

By employing STEM-EDS, Hentz et al. [96] have demonstrated that the darker domains in the dark field STEM image of a $\mathrm{MAPbI}_{3}$ perovskite lamella was enriched with iodide, which displayed an enhanced CL intensity. Moreover, Doherty and coworkers [68] performed low-dose scanning electron diffraction (SED) microscopy coupled with STEMEDS measurements to visualize local crystallography of heterogeneous grains and their correlation with trap clusters at grain boundaries, revealing the presence of an inhomogeneous grain with non-uniform $\mathrm{Br} / \mathrm{I}$ ratio (Figure 5F). Nevertheless, owing to the special requirements for sample preparation, concerns about electron beam induced damage, and accessibility issue, etc., as seen, only a few works dedicating to study halide segregation or local halide variations in MHPs using TEM or EDS-TEM have been reported thus far. 

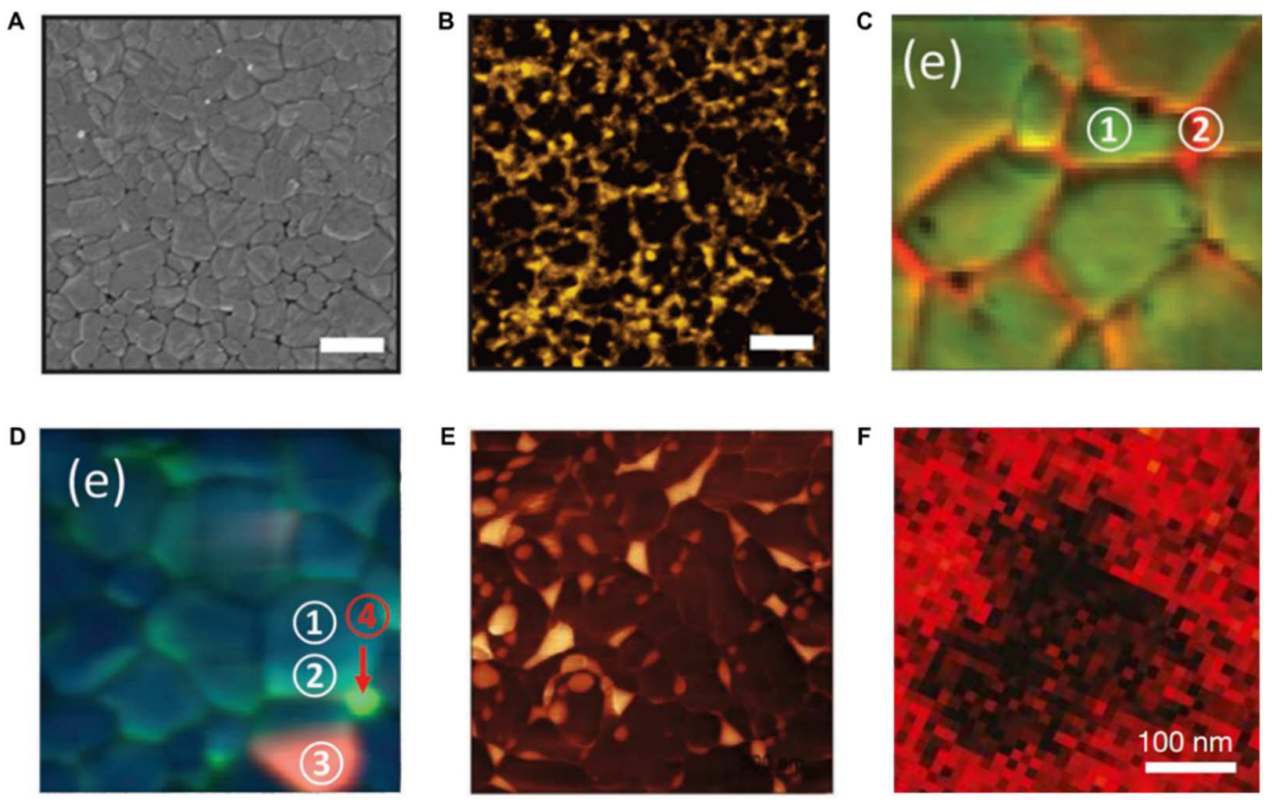

Figure 5. SEM image (A) and CL image (B) of a $\mathrm{MAPb}\left(\mathrm{I}_{0.1} \mathrm{Br}_{0.9}\right)_{3}$ perovskite film. Reproduced with permission [77]. Copyright 2017, American Chemical Society. (C) A superposition image of two $\mathrm{CL}$ images individually corresponding to the mixed phase emission and I-rich phase emission. The color code indicates the emission wavelength, that is orange domains suggest a longer fluorescence wavelength than the green domains. (D) A superposition image of two CL images. The pseudo color represents different fluorescence emission wavelengths. Reproduced with permission [91]. Copyright 2017, John Wiley \& Sons. (E) Phase contrast image obtained from AFM measurement of a $\mathrm{Cs}_{1.2} \mathrm{PbI}_{2} \mathrm{Br}_{1.2}$ film after continuous light soaking for $20 \mathrm{~h}$. Reproduced with permission [97]. Copyright 2021, John Wiley \& Sons. (F) Ratio $I(\mathrm{Br} \mathrm{K} \alpha) /(I(\mathrm{I} \mathrm{L} \alpha)+I(\mathrm{Br} \mathrm{K} \alpha))$ extracted from a STEMEDX map of a region of interest of a $\left(\mathrm{Cs}_{0.05} \mathrm{FA}_{0.78} \mathrm{MA}_{0.17}\right) \mathrm{Pb}\left(\mathrm{I}_{0.83} \mathrm{Br}_{0.17}\right)_{3}$ film. The dark region denotes iodide rich. Reproduced with permission [68]. Copyright 2020, Nature Publishing Group.

\subsection{Atomic Force Microscopy}

Atomic force microscopy (AFM) is an imperative and the most extensively used scanning probe microscopy (SPM) technique that has been well developed for imaging various material surfaces with nanoscale spatial resolution. This technique works based on the interaction forces between the atoms at sample surface and the probe tip, usually made of $\mathrm{Si}$, $\mathrm{SiO}_{2}$, or $\mathrm{Si}_{3} \mathrm{~N}_{4}$, which is attached to a cantilever [46]. When closing to a material surface, the tip and the cantilever can be deflected by forces such as mechanical contact forces, van der Waals forces, and electrostatic forces. Meanwhile, a displacement sensor measures the deflection of the cantilever with a sensitivity better than 0.1 nanometer by detecting the angle variation of a laser beam reflected from the backside of the cantilever, thus enabling to determine the specimen surface topography with resolutions in the order of a few nanometers [93]. Moreover, some derivative techniques, including kelvin probe force microscopy (KPFM), conductive-AFM (c-AFM), have been exploited. KPFM measures the contact potential difference (CPD) between the material surface and the tip, allowing to map the work function or surface potential $[97,98]$. While c-AFM method is an electrical model in the SPM family, which is used to study the conductivity and imaging electrical properties of the samples, such as charge transport and charge distribution at the nanoscale. AFM based techniques have been used to unveil variations of the topography, phase contrast, surface potential or photocurrent of the MHPs under illumination or electric field.

By utilizing photoconductive AFM (pc-AFM) coupled with PL imaging, Gomez et al. [99] have revealed that photocurrent changes initiated at the grain boundaries and gradually propagated into the grain interior of the as-prepared mixed cation and mixed halide perovskite films under continuous light soaking or applied voltage, which was 
performed in a dry air condition with relative humidity lower than $6 \%$. Additionally, they observed that this variation could be fully reversible in the grain interior, but unchanged at the boundaries due to severe degradation of the perovskite in these regions when the film was applied with an opposite voltage. More interestingly, targeting on a degraded area, the researchers found that the initial photocurrent could be fully recovered if the force on the tip was strong enough to penetrate several nanometers from the surface. Given this phenomenon, the authors inferred that ion migration and accumulation at the shallow surface occurred when the perovskite layer was subjected to light exposure and/or applied bias, which is in line with the work reported by Chen and coworkers using PL mapping, as discussed above [86]. Moreover, spatially resolved visualization of light-induced halide segregation over time in $\mathrm{CsPbI}_{2} \mathrm{Br}$ and $\mathrm{Cs}_{1.2} \mathrm{PbI}_{2} \mathrm{Br}_{1.2}$ films using in situ AFM was reported [97]. In this experiment, the illuminated region was continuously and repeatedly scanned to monitor any changes in topography, phase contrast and surface potential of the film. The $\mathrm{Cs}_{1.2} \mathrm{PbI}_{2} \mathrm{Br}_{1.2}$ film exhibits much higher stability performance than the $\mathrm{CsPbI}_{2} \mathrm{Br}$ counterpart owing to the stabilizing effect of the over-stoichiometric $\mathrm{CsBr}$ species. New phases firstly appearing at the grain boundaries were clearly observed in terms of the phase contrast images, as presented in Figure 5E. By determining and comparing the typical work functions of a suite of $\mathrm{CsPbI}_{3-x} \mathrm{~B}_{\mathrm{x}}$ based materials with different $\mathrm{I} / \mathrm{Br}$ ratios, the authors assign the segregated phases at the grain boundaries in $\mathrm{CsPbI}_{2} \mathrm{Br}$ and $\mathrm{Cs}_{1.2} \mathrm{PbI}_{2} \mathrm{Br}_{1.2}$ films to orthorhombic $\mathrm{CsPbI}_{3}$ and cubic $\mathrm{CsPbI}_{3}$, respectively. Therefore, the researchers conclude that I-rich phase is selectively expulsed from the bulk to grain boundaries under illumination, which matches previous studies well $[48,83,91,100]$.

\section{Mechanism of Halide Segregation in MHPs}

The underpinning mechanism of halide segregation in MHPs, especially induced by light irradiation, is the subject of great interest and intense debate in the perovskite community. Significant efforts have been made to get insight into the origin of this intriguing phenomenon. As a result, plentiful interpretations and models with multi-disciplinary background are proposed. However, an ultimate and comprehensive framework for unambiguous unveiling the mechanism and kinetics at the atomic scale has yet to be constructed. In this section, we will introduce and discuss the proposed mechanisms for photoinduced phase separation existing in the literature, which are classified into four categories, i.e., thermodynamic models, polaron/strain models, carrier gradient/carrier trapping models and redox models.

\subsection{Thermodynamic Models}

From a thermodynamic standpoint, Brivio et al. [101] calculated the free energy of $\mathrm{MAPb}\left(\mathrm{I}_{1-\mathrm{x}} \mathrm{Br}_{\mathrm{x}}\right)_{3}$ alloy as a function of the bromide content and temperature using firstprinciples total energy calculations, showing in Figure 6A. The curve is asymmetric at low temperature. While with the temperature increase, the shape of the curve becomes more symmetric. They further constructed a phase diagram of the $\mathrm{MAPb}\left(\mathrm{I}_{1-\mathrm{x}} \mathrm{Br}_{\mathrm{x}}\right)_{3}$ alloy based on the variation of Helmholtz free energy (Figure 6B). The obtained phase diagram suggests that the $\mathrm{MAPb}\left(\mathrm{I}_{1-\mathrm{x}} \mathrm{Br}_{\mathrm{x}}\right)_{3}$ compound features a miscibility gap below a critical temperature of $343 \mathrm{~K}$. It means that the alloys in this gap are miscible above the miscibility temperature and become unstable at lower temperature. Typically, the phase diagram displays a miscibility gap involving $0.3<\mathrm{x}<0.6$ for $\mathrm{MAPb}\left(\mathrm{I}_{1-\mathrm{x}} \mathrm{Br}_{\mathrm{x}}\right)_{3}$ at $300 \mathrm{~K}$, indicating that the compounds in this region are prone to spinodal decomposition and halide separation at room temperature. The authors considered that phase separation is spontaneous, even though this process could be very slow without external stimuli. Thus, light irradiation can expedite halide segregation by overcoming these kinetic barriers. This model can rationalize the existence of terminal $x$-value, but also predict an irreversible halide demixing process, which is obviously contradictory with the fact. Moreover, the conclusion that $\mathrm{MAPb}\left(\mathrm{I}_{1-\mathrm{x}} \mathrm{Br}_{\mathrm{x}}\right)_{3}(0.3<\mathrm{x}<0.6) \mathrm{MHPs}$ are unstable at room temperature is unreasonable because $\mathrm{MAPb}\left(\mathrm{I}_{1-\mathrm{x}} \mathrm{Br}_{\mathrm{x}}\right)_{3}$ with any $\mathrm{x}$ value can keep stable at such temperature [43]. 

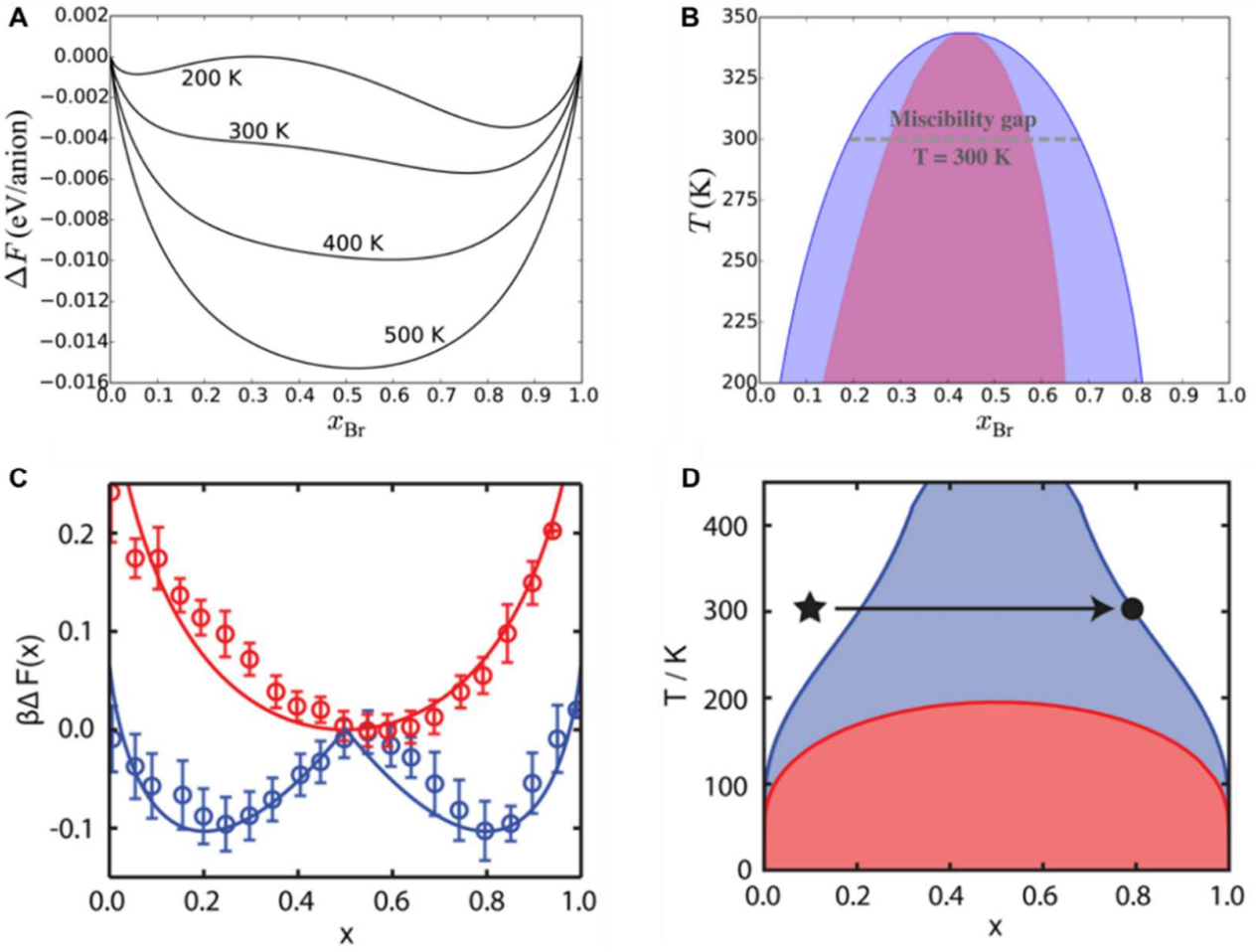

Figure 6. (A) Calculated Helmholtz free energy as a function of the bromide content and temperature of the $\mathrm{MAPb}\left(\mathrm{I}_{1-\mathrm{x}} \mathrm{Br}_{\mathrm{x}}\right)_{3}$ alloy composition using first-principles total energy calculations. (B) Predicted phase diagram of the $\mathrm{MAPb}\left(\mathrm{I}_{1-\mathrm{x}} \mathrm{Br}_{\mathrm{x}}\right)_{3}$ alloy. The purple and pink lines are the binodal and spinodal lines, respectively. Reproduced with permission [101]. Copyright 2016, American Chemical Society. (C) Free energies per unit cell for $\mathrm{MAPb}\left(\mathrm{I}_{\mathrm{x}} \mathrm{Br}_{1-\mathrm{x}}\right)_{3}$ with varying composition in the ground (red) and photoexcited (blue) states computed from molecular dynamics simulations (circles) and mean field theory (solid lines). (D) Mean field theory temperature-composition phase diagram in the ground (red) and photoexcited state (blue) with the path through the phase diagram from initial state (star) to demixed state (circle). Areas beneath the red and blue coexistence curves indicate segregated states. Reproduced with permission [77]. Copyright 2017, American Chemical Society.

Subsequently, by combining experimental and theoretical studies, Bischak and colleagues [77] provided a deep and comprehensive insight into photoinduced phase separation in MHPs. By using molecular dynamics simulations performed on $\mathrm{MAPb}\left(\mathrm{I}_{\mathrm{x}} \mathrm{Br}_{1-\mathrm{x}}\right)_{3}$, they found that elastic effects from lattice mismatch are much larger than specific chemical interactions, which leads to a demixing transition that depends strongly on strain. Furthermore, high spatial overlap between the lattice and a single-charge density distribution generates enough strain to be able to drive local phase separation at room temperature. Notably, with excess charge, the free energy as a function of composition appears two minima located at $X=0.2$ and $X=0.8$, respectively (Figure 6C). Previous PL studies demonstrate that the composition of segregated iodide-rich phase is fixed, approximate to $\mathrm{MAPb}\left(\mathrm{I}_{0.8} \mathrm{Br}_{0.2}\right)_{3}$, and regardless of the parent phase compositions, which verifies this prediction. Moreover, they established a thermodynamic model by modeling the phase segregation using a Landau-Ginzburg Hamiltonian with linear coupling between strain and composition fields and employing a semiclassical description of the excess charge. Free energies per unit cell for $\mathrm{MAPb}\left(\mathrm{I}_{x} \mathrm{Br}_{1-\mathrm{x}}\right)_{3}$ with varying composition in the ground and photoexcited states predicted by this theory are in good agreement with the results obtained from molecular dynamics simulations. They constructed the temperature-composition phase diagram of $\mathrm{MAPb}\left(\mathrm{I}_{\mathrm{x}} \mathrm{Br}_{1-\mathrm{x}}\right)_{3}$ for both ground- and photoexcited states by adopting mean field approximation (Figure 6D). Besides, the extent of halide demixing with respect to electron-phonon coupling and temperature was mapped based on mean field theory in the photoexcited state. We can see that the thermodynamic model is capable of rationalizing 
a wide range of experimental observations. This model highlights that the photogenerated charge carriers localized in the I-rich clusters are essential for stabilizing the segregated I-rich phase through electron-phonon coupling. The reversibility of halide segregation in the dark and the existence of terminal $x$ value are predicted. Besides, decreasing electronphonon coupling in the system is expected to suppress halide demixing, which can explain why doping the perovskites with Cs cation enables to enhance their photostability. Since the kernel of this model is invoking local lattice deformation induced by photogenerated charges as the origin of halide segregation, this model is thus also regarded as a polaron or strain model, which will be further discussed in the next subsection.

On the other hand, a type of thermodynamic models based on band gap difference between the parent and I-rich phases which leads to photocarriers funneling to lowering the free energy are proposed [102-104]. Draguta et al. [102] conclude that bandgap reduction in I-rich domains is responsible for the driving force that leads to halide segregation. I-rich phases feature lower valence band edges and nearly isoenergetic conduction band edges with respect to the mixed phase, thus favoring photogenerated carriers diffusing into I-rich regions and then recombining radiatively. Reducing carrier diffusion lengths and lowering illumination intensity contribute to suppressing halide segregation. To verify the second prediction, the authors compared the light-induced separation behavior of $\mathrm{CsPb}\left(\mathrm{I}_{0.5} \mathrm{Br}_{0.5}\right)_{3}$ thin films and nanocrystal-based films. They attributed the much enhanced photostability of the nanocrystal-based films to the reduction in carrier diffusion lengths. However, we note that it could be unpersuasive and the diffusion lengths do not directly dictate phase segregation. The underlying reason for this case, instead, from the perspective of thermodynamic or rather nucleation theory, is that the interfacial energy cost of forming new interface in nanocrystals becomes significant, thus removing the thermodynamic driving force for phase separation [105-109]. Moreover, assuming a perovskite film with high defect density, the carrier diffusion lengths are short, while photoinduced halide demxing effect would be pronounced. Whereas, this analytical model has made success in explaining the existence of excitation intensity threshold, the observed non-linear intensity dependencies in kinetic rate constants and self-limited growth of I-rich domains.

Recently, the same group [103] further supported their band gap difference based thermodynamic model. It is worth noting that a distinguishing feature of this model compared with thermodynamic miscibility gap models [77,101], as claimed, does not exist free energy minimum value varied with temperature. This is due to the free energy primarily arises from band gap difference between I-rich phase and mixed phase. With this regard, they examined the impact of temperature on the terminal $x$ value of segregated I-rich phase using $\mathrm{PL}$ measurements. In the case of $\mathrm{MAPb}\left(\mathrm{I}_{0.5} \mathrm{Br}_{0.5}\right)_{3}$ thin film, the PL emission corresponding to the segregated I-rich phase at $185 \mathrm{~K}$ show a small red-shift $(\sim 20 \mathrm{meV})$ as compared with the emission wavelength at $295 \mathrm{~K}$. As for $\mathrm{MA}_{0.5} \mathrm{Cs}_{0.5} \mathrm{~Pb}\left(\mathrm{I}_{0.5} \mathrm{Br}_{0.5}\right)_{3}$ thin film, this variation was smaller. Thus, the authors concluded that the terminal $x$ value did not change significantly with temperature, supporting band gap difference as the dominant contributor to free energy that drives photosegregation. Almost at the same time, Chen et al. [104] reported a unified thermodynamic theory for photoinduced halide segregation based on the band gap difference between the parent phase and the I-rich phase. The photogenerated carriers lower their free energy by funneling to the I-rich inclusions. They adopted Boltzmann statistics model to determine the distribution of photogenerated electrons or holes over the two phases in the system at equilibrium state. Using this theory, they have constructed the phase diagrams for MA-, FA-, Cs-, MA/Cs-, and FA/Cs-based MHPs in the dark and under illumination and shown the dependence of threshold photocarrier density for halide segregation on $\mathrm{Br}$ concentration and temperature. This theory is capable of accounting for many experimental findings, such as a highly composition and temperature dependent illumination intensity threshold for halide segregation, the stabilization effect of alloying Cs cation into the perovskites, and flash formation of pure iodide clusters at the initial stage of halide demixing [72,110]. However, this model predicts a boundless phase separation behavior with the increase of light intensity, which is contradictory with the 
observed light-induced halide remixing phenomenon under sufficient high light intensity reported by Mao et al. [111] and Guo et al. [112].

\subsection{Polaron/Strain Models}

The formation of polarons, originating from the coupling of free carriers with the lattice, has also been proposed as a promising mechanism to explain the photoinduced halide segregation effect in MHPs [75,77,109,111,112]. Owing to the soft nature of the inorganic lattice frameworks with small bulk modulus, the photogenerated charge carriers could induce sizable local lattice deformation, bringing excessive strain energy in the systems. Thus, the releasing of strain energy through the redistribution of halide ion leads to ultimate phase separation [109]. Besides, there has another report which considers that localized strain arising from crystal growth process could also play an important role in light-induced halide segregation [57].

Bischak et al. [77] were the first to propose the polaron model to explain the origin of halide segregation, which has been partially discussed above. Figure 7A illustrates the generation of free carriers upon light photoexcitation and the formation and trapping of polarons. Owing to the small exciton binding energies in MHPs, photogenerated excitons could rapidly dissociate and form free carriers. These charges deform the surrounding lattice through electron-phonon coupling and thus change the free energy landscape of the system. They suggest that halide demixing would happen to reduce free energy when the local lattice strain is sufficiently large, as diagramed in Figure 7B. This model highlights that the segregated I-rich inclusions could only maintain stable with the presence of continuously generated charge carriers. In this context, some key experimental observations of halide segregation can be rationalized, including the reversibility [31], light intensity dependence of demixing rate [57,102], self-limited growth of I-rich domains [77,102], preferentially appearing at the regions featuring high defect density $[59,63,75]$, the existence of light intensity threshold $[66,102,104,113,114]$, stabilization effect of Cs cation $[67,75,77,78,80-82,115]$, suppressed phase separation under the circumstance of fast charge extraction $[71,116,117]$, etc. Some articles deem that the polaron model does not dictate an excitation intensity threshold and thus even the lowest excitation should initiate segregation [38,42]. However, we think that such a view is unreasonable because the loss of photogenerated charge carriers due to the trap-state mediated non-radiative recombination should be taken into consideration. Besides, as said above, only when the local lattice deformation induced by free carriers is sufficiently large such that the free energy of the system turns into positive, halide demixing could take place.

Subsequently, Bischak et al. [75] deem that photoinduced phase segregation is an intrinsic property of MHPs by examining the halide demixing behavior in monocrystalline perovskite nanoplates. Furthermore, suppressed light-induced halide segregation upon partially substituting MA cation with Cs cation was observed, supporting the polaron model because the presence of Cs cation can reduce electron-phonon coupling strength in the perovskites due to lattice stiffening. Wang and coworkers [109] have also invoked the polaron model combined with thermodynamic model based on nucleation theory to explain the origin of the excellent photostability of the $\mathrm{CsPb}\left(\mathrm{Br}_{x} \mathrm{I}_{1-x}\right)_{3}$ nanoparticles, which are confined in the $\mathrm{Cs}_{4} \mathrm{~Pb}\left(\mathrm{Br}_{x} \mathrm{I}_{1-\mathrm{x}}\right)_{6}$ host matrix. The as-prepared composite thin films with a host-guest structure could even withstand an ultra-high light intensity of $440 \mathrm{~W} \mathrm{~cm}^{-2}$, that is 4400 suns illumination, and remain PL emission stable after $4 \mathrm{~h}$ of irradiation if the $\mathrm{CsPb}\left(\mathrm{Br}_{x} \mathrm{I}_{1-\mathrm{x}}\right)_{3}$ nanocrystals feature a $\mathrm{x}$ value no more than 0.6 . The average size of the $\mathrm{CsPb}\left(\mathrm{Br}_{x} \mathrm{I}_{1-\mathrm{x}}\right)_{3}$ nanocrystals is about $7.5 \mathrm{~nm}$. They think that photoinduced polarons bring in excessive strain energy due to the deformation of the lattice, contributing to the increase of Gibbs free energy. While in the case of such small nanocrystals embedded in the matrix, the cohesive energy would dominate over the photoinduced strain energy, enabling a highly stable homogenous phase even under extremely intensive illumination. From a nucleation theory standpoint, I-rich domains could never nucleate out of the parent phase 
to reach a certain critical size because the average domain size of the mixed phase is smaller than the critical size, thus halide segregation is energy unfavorable.

A

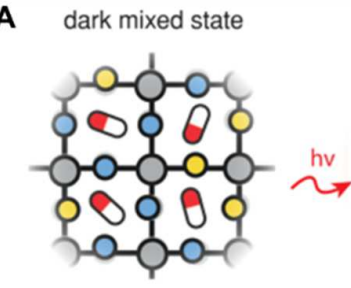

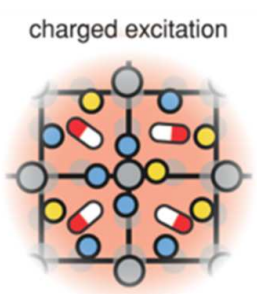

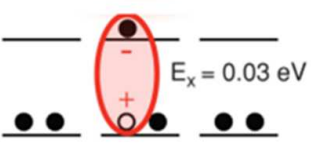

-

$E_{\text {gap }}=2.25 \mathrm{eV}$

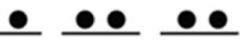

누우
C

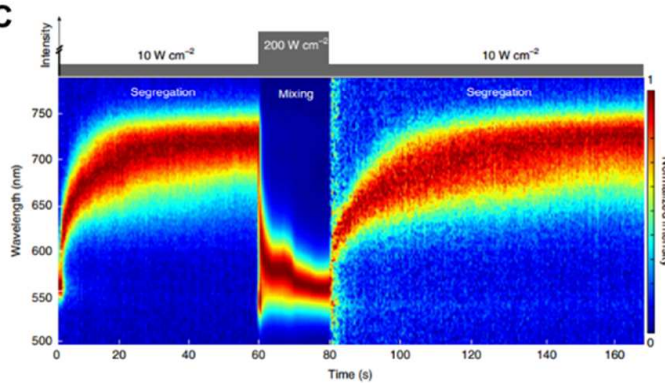

demixed trapped state

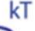

kT
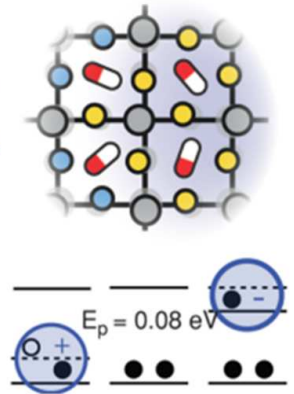

B

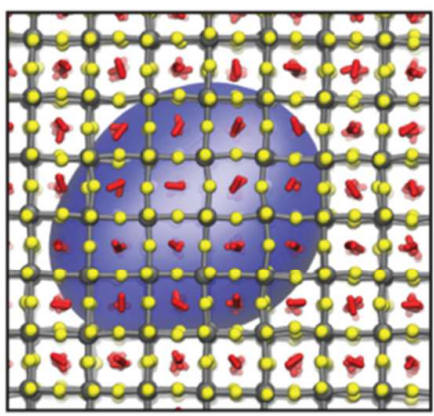

D

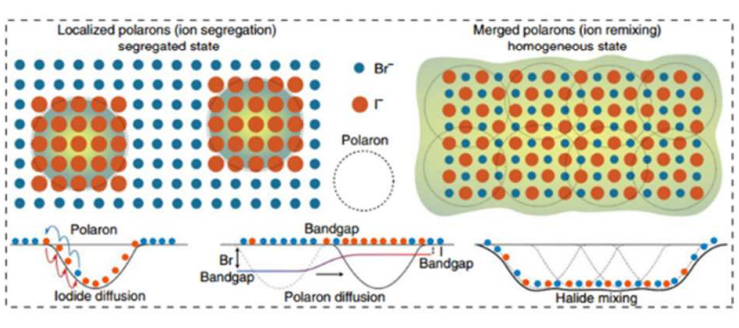

Figure 7. (A) Diagram of photoinduced polaron trapping and associated energy scales associated with phase separation. (B) Snapshot of the $99 \%$ isosurface of excess charge density taken from the molecular dynamics simulation. Reproduced with permission [77]. Copyright 2017, American Chemical Society. (C) Normalized PL emission spectra over time of a $\mathrm{MAPb}\left(\mathrm{Br}_{0.8} \mathrm{I}_{0.2}\right)_{3}$ monocrystalline microplate under illumination with two different light intensity using a laser as the light source $\left(10 \mathrm{~W} \mathrm{~cm}^{-2}\right.$ and $200 \mathrm{~W} \mathrm{~cm}^{-2}$ ). (D) Schematic illustration of halide ion distributions for a $\mathrm{MAPb}\left(\mathrm{Br}_{0.8} \mathrm{I}_{0.2}\right)_{3}$ perovskite lattice in response to low carrier density (left) and high carrier density (right). The proposed lattice model includes three driving mechanisms: (1) iodide diffusion along polaron strain gradients, (2) polaron diffusion along iodide concentration gradients and (3) halide mixing in the absence of strong strain gradients. Reproduced with permission [111]. Copyright 2021, Nature Publishing Group.

Recently, a very interesting finding that halide remixing under sufficiently high light intensities has been reported [111]. As shown in Figure 7C, when the light-induced segregated perovskite film is subjected to ultra-high illumination irradiation $\left(200 \mathrm{~W} \mathrm{~cm}^{-2}\right)$, the halide species would be remixed. The researchers elucidated the underlying mechanism based on the polaron model, as illustrated in Figure 7D. In the case of low illumination intensity, photogenerated carriers induced strain gradient favors halide demixing. Whereas, under sufficiently strong light irradiation, the strain gradient can be significantly reduced, owing to the polarons overlap or merging, thus halide remixing occurs driven by entropy. Similar photoinduced halide demixing and remixing phenomenon has also been reported by another work [112]. In this contribution, the authors found that phase transformations from halide mixing to demixing and then to remixing could always occur in polycrystalline $\mathrm{CsPb}\left(\mathrm{Br}_{\mathrm{x}} \mathrm{I}_{1-\mathrm{x}}\right)_{3}$ films under continuous light soaking. Intriguingly, the film after halide remixing is immune to light-induced halide segregation. Meanwhile, nanoscale cracks were observed in the perovskite film, which were thought to be the consequence of strain release. This finding can be regarded as a concrete and direct evidence for supporting the polaron/strain model responsible for photosegreagtion. In addition, there is another study which points out that the strain generated from film fabrication process should play a significant role in facilitating light-induced phase separation [57]. The authors demonstrate an averaging $0.3 \%$ strain in $\mathrm{MAPb}\left(\mathrm{I}_{1-\mathrm{x}} \mathrm{Br}_{\mathrm{x}}\right)_{3}$ polycrystalline perovskite film is much larger 
than that in single crystal $(\sim 0.0004 \%)$, which prefers to concentrate at the grain boundaries. Notably, they conclude that the driving force might deriving from bandgap reduction or polaron is not enough to induce halide segregation within the strain-activated regime if without the synergy of strain.

\subsection{Carrier Gradient/Carrier Trapping Models}

Another category of the proposed mechanisms, as has been reviewed and discussed in previous articles [38,42], invokes carrier gradient/carrier trapping as the internal causes for light-induced halide segregation [61-63]. These types of empirical models highlight the essential role of perovskite defects in determining the photoinduced halide segregation.

Given the excellent light absorption properties of the lead halide perovskites, Barker et al. [61] hold the view that internal gradient in carrier generation rate under illumination along the vertical direction of MHP films may be responsible for halide demixing. As for how carrier gradient could lead to halide species migration and segregation, the authors consider that it could be due to the photoinduced strain gradient or the thermalization energy from hot carriers near the illuminated surface. To verify their model, the researchers have compared the photostability of thick perovskite films $(280 \mathrm{~nm})$ and thin ones $(70 \mathrm{~nm})$ with identical composition. Interestingly, the thin films exhibited much better photostability than the thick films, which could ascribe to a more homogeneous carrier generation throughout the thin perovskite layers. Particularly, they demonstrate that halide ion segregation takes place via halide defects, or rather halide vacancies, resulting in I-rich inclusions close to the illuminated side of the film, owing to a slower hopping rate of iodide compared with bromide ions away from the film surface. Notably, suppressed photosegregation by adding over-stoichiometric halide species in the precursor solution confirms the important role of vacancy defects in influencing halide ion migration and demixing $[60,61,97]$.

Subsequently, McGehee group [62] proposed that carrier trapping by surface defects could induce the formation of electric fields. The as-formed electric fields, pointing toward the surface, lead to halide vacancies migration and accumulation near the perovskite surface, which is likely to be equivalent to a preferred drift of bromide ions away from the surface, thus resulting in halide demixing and I-rich phase emerging at these regions. In this study, they suggested that reducing surface defects or preventing positive charge cumulation at the surface would benefit the suppression of photoinduced halide segregation, which indeed make sense, and the latter has been underpinned by other works [100,116,118]. However, we note that any explicit evidences of the electric fields existing near the surface and bromide migrating away from the surface were not presented in this work. Certainty about which kind of vacancies, bromide or iodide, are dominant in the MHP films under light soaking has remained debatable [119,120].

Similarly, Knight et al. [63] also hold the view that halide segregation is driven by electric fields in the film, which are generated between the trapped electrons in defect states and the confined holes in I-rich regions, due to funneling effect. Following this logic, the authors propose such a scenario that an electric field stimulates halide demixing, which, in turn, promotes charge separation, thus increasing the strength of the electric field, that is, forming a positive feedback loop between the electric field and halide segregation, until reaching a maximally segregated state. In light of this mechanism, they have explained why atmospheric environments display significant impacts on halide segregation dynamics. They believe that it is derived from the variation of trap-state density in perovskite films under different atmospheric environments over time. In this way, they have shown that encapsulation of $\mathrm{MAPb}\left(\mathrm{Br}_{0.5} \mathrm{I}_{0.5}\right)_{3}$ films with a poly(methyl methacrylate) (PMMA) coating enables halide segregation to be fully reversible and repeatable.

\subsection{Redox Models}

Recently, a kind of redox models for halide segregation centered on holes induced iodide oxidation and migration of oxidized species have been proposed and developed $[45,97,100,116]$. Since 2018, several works correlated with hole trapping leading 
to expulsion of iodide from the lattice in the form of neutral iodine have been successively reported $[83,100,116,118,120]$.

Initially, to unveil the underlying mechanism of photoinduced ion conductivity enhancement in perovskite films, Kim et al. [120] performed one of a simple experiment that immersing $\mathrm{MAPbI}_{3}$ films in toluene with or without light irradiation and recording the in situ ultraviolet-visible (UV-vis) absorption signal of $\mathrm{I}_{2}$ in toluene over time. They found that the amount of iodine in toluene was significantly increased under illumination, suggesting light-induced expulsion of iodine from the perovskite lattice. They suggested that this is due to iodide oxidation in the presence of photogenerated holes, resulting in the formation of interstitial neutral iodine and iodide vacancies.

Moreover, Kamat and coworkers found that selectively electrochemical injection of holes into MHP films could lead to iodide expulsion and halide segregation, as diagramed in Figure 8A [100]. Additionally, they also observed that the perovskite films prepared on the $\mathrm{TiO}_{2}$ electron transporting layers exhibited pronounced halide segregation when subjected to laser irradiation (Figure 8B), while, intriguingly, the perovskite films deposited on the $\mathrm{ZrO}_{2}$ layers remained stable within a same period of time. Furthermore, photosegregation was suppressed upon coating a hole transporting layer [116]. In this context, the authors believe that hole trapping at the iodide site gives rise to iodide species moving toward grain boundaries via halide vacancies in the form of iodine and/or triiodide ions, thus leading to halide segregation. That is to say, hole accumulation and trapping are responsible for phase separation.

A

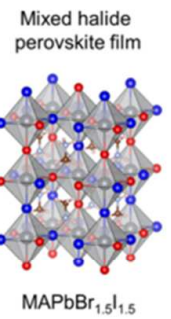

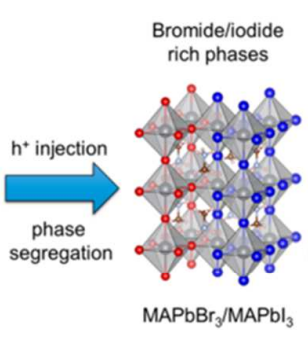
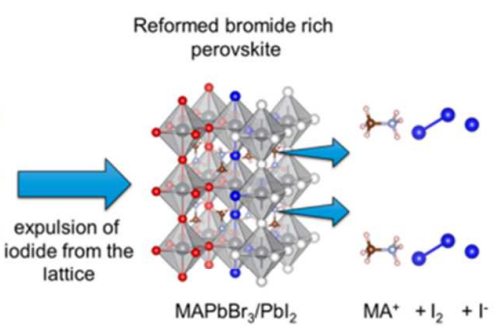

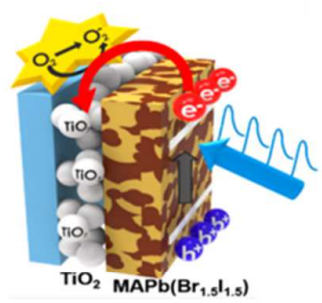

C

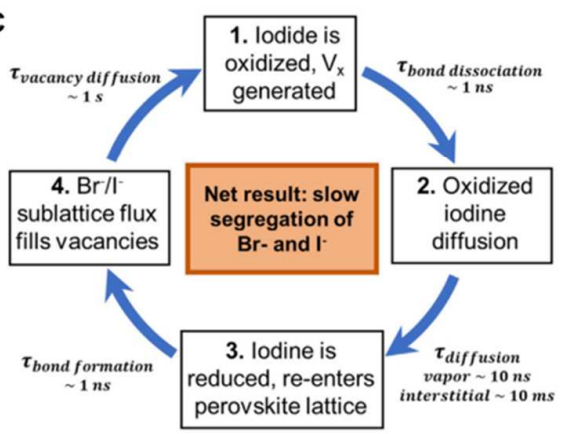

D

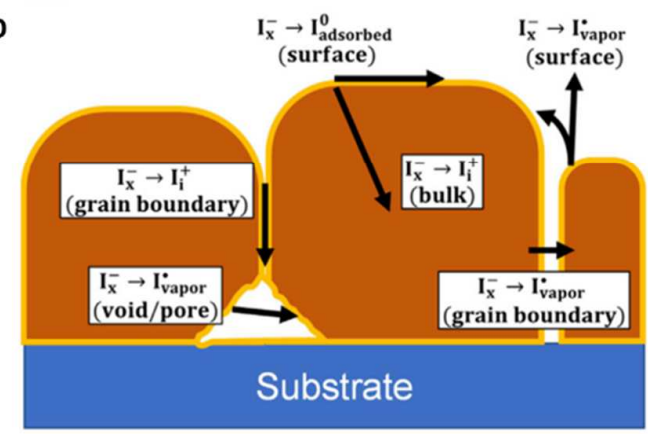

Figure 8. (A) Illustration of halide demixing and iodide expulsion induced by hole injection into the MHP lattice. Reproduced with permission [100]. Copyright 2019, American Chemical Society. (B) Schematic illustration of $\mathrm{TiO}_{2}$-assisted halide segregation in MHP film under pulsed laser excitation. Reproduced with permission [116]. Copyright 2020, American Chemical Society. (C) Diagram of a series of microscopic events (including timescales) that a halide experiences during its oxidation-mediated redistribution. (D) Possible mechanisms and pathways for vertical and lateral mass transport of the oxidized iodine species. Reproduced with permission [45]. Copyright 2021, Elsevier.

Subsequently, Frolova et al. [97] claimed that reversible $\mathrm{Pb}^{2+} / \mathrm{Pb}^{0}$ and $\mathrm{I}^{-} / \mathrm{I}^{3-}$ redox chemistry drives the photoinduced halide segregation in inorganic MHPs, supporting the model proposed by Kamat and colleagues [100,116]. On the basis of the results obtained from in-situ AFM measurements, they deem that the segregated I-rich phase at grain 
boundaries is derived from light triggered photochemical processes, which results in the formation of $\mathrm{I}^{3-}$ and $\mathrm{Pb}^{0}$ species. These species would be expelled to the perovskite surface and grain boundaries through halide vacancies or locally produced in these regions. Then they undergo reversible redox processes and convert into perovskite phase again. According to this explanation, the content of $\mathrm{Pb}^{0}$ species in perovskites should be much higher under light soaking than in the dark, while such evidence is lacking.

Very recently, on the basis of previous works, Kerner and coworkers [45] have developed this type of model and expounded it in great detail. They highlight the key role of photoelectrochemistry processes in halide segregation, and iodide oxidation induced by photogenerated holes appears to initiate the halide demixing event. The authors consider that all oxidized halide species are much more mobile than halide ions at the lattice sites, and any factors causing iodide oxidation rate discrepancy, including defects, heterogeneity, film thickness, etc., could promote halide segregation by generating local gradients in the concentration of oxidized halide species. Figure 8C,D illustrate their electrochemical perspective where preferential redox of iodide leads to halide segregation in MHPs and the transport pathways of the oxidized species in perovskite films. Notably, they emphasize that the valence band maximum (VBM) of the perovskites should be deep enough, thus enables the photogenerated holes to activate relevant iodide oxidation pathways. In this regard, it necessitates a threshold bromide content for the perovskite compositions, might rationalizing why halide segregation only takes place within specific $x$ value ranges as observed in the experiments $[31,57,121]$. Additionally, vapor phase ( $\mathrm{I}_{2}$ vapor) transport is thought to be an important mass transport form of the oxidized iodine species in the perovskites, which thus is capable of explaining why the reversibility of halide segregation is undermined under vacuum condition, possibly due to the loss of iodide component [63]. Overall, the redox model developed here provides an alternative perspective to reinterpret a wide range of experimental observations in regard to halide segregation reported in the literature.

\section{Summary and Outlook}

In this review, we have systematically summarized the research efforts on visualized observation of halide segregation under external stimuli, especially light soaking. Microscopy techniques, including PL mapping, CL imaging, TEM, EDS, AFM, have been utilized to probe local halide demixing behaviors at the nanoscale or microscale. Among them, PL mapping is the most widely used spatially resolved method to imaging phase segregation of MHPs, owing to its noninvasive, nondestructive characteristics and desirable operational flexibility. There is a general consensus that highly defective regions of the perovskites are more susceptible to halide segregation, where the segregated I-rich phase aggregate, for instance, the grain boundaries. However, halide demixing observed at monocrystalline MHPs demonstrated that grain boundaries are not necessary for it to happen. Instead, edge and surface states are shown to play a critical role in altering the phase segregation. Additionally, a consensus has yet to be reached on whether halide demixing repeatedly appears at the same locations in different light on/off cycles, necessitating further clarification.

After that, mechanisms existing in the literature for explaining the origin and evolution of photoinduced halide segregation, involving thermodynamic models, polaron/strain models, carrier gradient/carrier trapping models and redox models, are comprehensively introduced and discussed. These established models promote the community's understanding of this effect. Currently, however, no one has been able to rationalize all experimental observations. It is quite clear now that nonequilibrium charge carriers (photogenerated, electron beam induced or electrochemically injected), the content of bromide, and crystal defects are imperative for phase segregation. While, whether it is an intrinsic property of this type of materials or not is still under debate. That is, could halide segregation still happen in a perfect monocrystalline MHP crystal without any defect? Thermodynamic models stand photoinduced phase segregation is an intrinsic property of the MHPs [77,101]. 
Whereas, since carrier gradient/carrier trapping models as well as redox models are established based on the inhomogeneity and crystal defects of the materials, thus halide demixing phenomenon is expected to disappear in a perfect MHP crystal in view of the two types of models $[45,61-63,97,100,116]$. Which prediction is correct is thus far unclear.

Moreover, there still exists some other open questions that general consensuses are still lacking. For example, what is the exact mechanism behind $\mathrm{Cs}$ cation and $\mathrm{Cl}$ anion improving the resistance to halide segregation of the perovskites $[75,77,82,104,113,115,118,122,123]$ ? What is the real reason for the decreased reversibility of light-induced halide demixing and remixing in vacuum observed in experiments $[45,63]$ ? What directly dictate halide segregation? Is it due to the migration of iodide species, or bromide species, or both? Which one moves faster? Does there exist direct halide substitution or exchange processes between iodide and bromide ions during halide segregation? Which kind of mobile species dominate photoinduced phase separation? Is halide ions or oxidized halide species $[45,61,62,83,86,97,100,120,124]$ ? Clarifying these questions is conducive to constructing a definitive model for photoinduced halide segregation. Overall, we believe that through collaboration across many scientific disciplines, the painted veil of light-induced phase separation in MHPs will be completely unveiled, thus enabling the community to precisely inhibit or utilize it for a broad range of applications in the future.

Author Contributions: Conceptualization, R.W. and L.T.; writing-original draft preparation, L.T.; writing-review and editing, R.W., J.X. and L.T. All authors have read and agreed to the published version of the manuscript.

Funding: J.X. acknowledges the startup funding from Zhejiang University and the financial support by Shanxi-Zheda Institute of Advanced Materials and Chemical Engineering (2021SZ-FR006). R.W. acknowledges the startup funding from Westlake University. J.X. and R.W. acknowledge the grant (LD22E020002) by Natural Science Foundation of Zhejiang Province of China.

Institutional Review Board Statement: Not applicable.

Informed Consent Statement: Not applicable.

Data Availability Statement: Not applicable.

Conflicts of Interest: The authors declare no conflict of interest.

\section{References}

1. Kojima, A.; Teshima, K.; Shirai, Y.; Miyasaka, T. Organometal halide perovskites as visible-light sensitizers for photovoltaic cells. J. Am. Chem. Soc. 2009, 131, 6050-6051. [CrossRef] [PubMed]

2. $\quad$ Kim, H.S.; Lee, C.R.; Im, J.H.; Lee, K.B.; Moehl, T.; Marchioro, A.; Moon, S.J.; Humphry-Baker, R.; Yum, J.H.; Moser, J.E.; et al. Lead iodide perovskite sensitized all-solid-state submicron thin film mesoscopic solar cell with efficiency exceeding $9 \%$. Sci. Rep. 2012, 2, 591. [CrossRef] [PubMed]

3. Lee, M.M.; Teuscher, J.; Miyasaka, T.; Murakami, T.N.; Snaith, H.J. Efficient hybrid solar cells based on meso-superstructured organometal halide perovskites. Science 2012, 338, 643-647. [CrossRef] [PubMed]

4. Yang, W.S.; Park, B.W.; Jung, E.H.; Jeon, N.J.; Kim, Y.C.; Lee, D.U.; Shin, S.S.; Seo, J.; Kim, E.K.; Noh, J.H.; et al. Iodide management in formamidinium-lead-halide-based perovskite layers for efficient solar cells. Science 2017, 356, 1376-1379. [CrossRef] [PubMed]

5. Jiang, Q.; Zhao, Y.; Zhang, X.; Yang, X.; Chen, Y.; Chu, Z.; Ye, Q.; Li, X.; Yin, Z.; You, J. Surface passivation of perovskite film for efficient solar cells. Nat. Photonics 2019, 13, 460-466. [CrossRef]

6. Yoo, J.J.; Seo, G.; Chua, M.R.; Park, T.G.; Lu, Y.; Rotermund, F.; Kim, Y.K.; Moon, C.S.; Jeon, N.J.; Correa-Baena, J.P.; et al. Efficient perovskite solar cells via improved carrier management. Nature 2021, 590, 587-593. [CrossRef] [PubMed]

7. Min, H.; Lee, D.Y.; Kim, J.; Kim, G.; Lee, K.S.; Kim, J.; Paik, M.J.; Kim, Y.K.; Kim, K.S.; Kim, M.G.; et al. Perovskite solar cells with atomically coherent interlayers on $\mathrm{SnO}_{2}$ electrodes. Nature 2021, 598, 444-450. [CrossRef]

8. Park, N.G.; Zhu, K. Scalable fabrication and coating methods for perovskite solar cells and solar modules. Nat. Rev. Mater. 2020, 5, 333-350. [CrossRef]

9. Bu, T.; Li, J.; Li, H.; Tian, C.; Su, J.; Tong, G.; Ono, L.K.; Wang, C.; Lin, Z.; Chai, N.; et al. Lead halide-templated crystallization of methylamine-free perovskite for efficient photovoltaic modules. Science 2021, 372, 1327-1332. [CrossRef]

10. Chen, S.; Dai, X.; Xu, S.; Jiao, H.; Zhao, L.; Huang, J. Stabilizing perovskite-substrate interfaces for high-performance perovskite modules. Science 2021, 373, 902-907. [CrossRef] 
11. Liu, Z.; Qiu, L.; Ono, L.K.; He, S.; Hu, Z.; Jiang, M.; Tong, G.; Wu, Z.; Jiang, Y.; Son, D.-Y.; et al. A holistic approach to interface stabilization for efficient perovskite solar modules with over 2000-hour operational stability. Nat. Energy 2020, 5, 596-604. [CrossRef]

12. Wang, R.; Xue, J.; Wang, K.L.; Wang, Z.K.; Luo, Y.; Fenning, D.; Xu, G.; Nuryyeva, S.; Huang, T.; Zhao, Y.; et al. Constructive molecular configurations for surface-defect passivation of perovskite photovoltaics. Science 2019, 366, 1509-1513. [CrossRef] [PubMed]

13. Quan, L.N.; Rand, B.P.; Friend, R.H.; Mhaisalkar, S.G.; Lee, T.W.; Sargent, E.H. Perovskites for Next-Generation Optical Sources Chem. Rev. 2019, 119, 7444-7477. [CrossRef] [PubMed]

14. Zhao, B.; Bai, S.; Kim, V.; Lamboll, R.; Shivanna, R.; Auras, F.; Richter, J.M.; Yang, L.; Dai, L.; Alsari, M.; et al. High-efficiency perovskite-polymer bulk heterostructure light-emitting diodes. Nat. Photonics 2018, 12, 783-789. [CrossRef]

15. Chu, S.; Chen, W.; Fang, Z.; Xiao, X.; Liu, Y.; Chen, J.; Huang, J.; Xiao, Z. Large-area and efficient perovskite light-emitting diodes via low-temperature blade-coating. Nat. Commun. 2021, 12, 147. [CrossRef]

16. Schanze, K.S.; Kamat, P.V.; Yang, P.; Bisquert, J. Progress in Perovskite Photocatalysis. ACS Energy Lett. 2020, 5, $2602-2604$. [CrossRef]

17. Guan, Z.; Wu, Y.; Wang, P.; Zhang, Q.; Wang, Z.; Zheng, Z.; Liu, Y.; Dai, Y.; Whangbo, M.-H.; Huang, B. Perovskite photocatalyst $\mathrm{CsPbBr}_{3-x} \mathrm{I}_{\mathrm{x}}$ with a bandgap funnel structure for $\mathrm{H}_{2}$ evolution under visible light. Appl. Catal. B 2019, 245, 522-527. [CrossRef]

18. Wei, H.T.; Fang, Y.J.; Mulligan, P.; Chuirazzi, W.; Fang, H.H.; Wang, C.C.; Ecker, B.R.; Gao, Y.L.; Loi, M.A.; Cao, L.; et al. Sensitive X-ray detectors made of methylammonium lead tribromide perovskite single crystals. Nat. Photonics 2016, 10, 333-339. [CrossRef]

19. Tsai, H.H.; Liu, F.Z.; Shrestha, S.; Fernando, K.; Tretiak, S.; Scott, B.; Vo, D.T.; Strzalka, J.; Nie, W.Y. A sensitive and robust thin-film x-ray detector using 2D layered perovskite diodes. Sci. Adv. 2020, 6, aay0815. [CrossRef]

20. Dong, H.; Zhang, C.; Liu, X.; Yao, J.; Zhao, Y.S. Materials chemistry and engineering in metal halide perovskite lasers. Chem. Soc. Rev. 2020, 49, 951-982. [CrossRef]

21. Zhang, Q.; Shang, Q.; Su, R.; Do, T.T.H.; Xiong, Q. Halide Perovskite Semiconductor Lasers: Materials, Cavity Design, and Low Threshold. Nano Lett. 2021, 21, 1903-1914. [CrossRef] [PubMed]

22. She, X.J.; Chen, C.; Divitini, G.; Zhao, B.D.; Li, Y.; Wang, J.Z.; Orri, J.F.; Cui, L.S.; Xu, W.D.; Peng, J.; et al. A solvent-based surface cleaning and passivation technique for suppressing ionic defects in high-mobility perovskite field-effect transistors. Nat. Electron. 2020, 3, 694-703. [CrossRef]

23. Senanayak, S.P.; Abdi-Jalebi, M.; Kamboj, V.S.; Carey, R.; Shivanna, R.; Tian, T.; Schweicher, G.; Wang, J.; Giesbrecht, N.; Di Nuzzo, D.; et al. A general approach for hysteresis-free, operationally stable metal halide perovskite field-effect transistors. Sci. Adv. 2020, 6, eaaz4948. [CrossRef]

24. Liu, T.; Zhao, X.; Li, J.; Liu, Z.; Liscio, F.; Milita, S.; Schroeder, B.C.; Fenwick, O. Enhanced control of self-doping in halide perovskites for improved thermoelectric performance. Nat. Commun. 2019, 10, 5750. [CrossRef] [PubMed]

25. Xu, J.; Li, X.; Xiong, J.; Yuan, C.; Semin, S.; Rasing, T.; Bu, X.H. Halide Perovskites for Nonlinear Optics. Adv. Mater. 2020, 32, e1806736. [CrossRef] [PubMed]

26. Privitera, A.; Righetto, M.; Cacialli, F.; Riede, M.K. Perspectives of Organic and Perovskite-Based Spintronics. Adv. Opt. Mater. 2021, 9, 2100215. [CrossRef]

27. Dunlap-Shohl, W.A.; Zhou, Y.; Padture, N.P.; Mitzi, D.B. Synthetic Approaches for Halide Perovskite Thin Films. Chem. Rev. 2019, 119, 3193-3295. [CrossRef]

28. Kim, J.Y.; Lee, J.W.; Jung, H.S.; Shin, H.; Park, N.G. High-Efficiency Perovskite Solar Cells. Chem. Rev. 2020, $120,7867-7918$. [CrossRef]

29. Lai, M.; Obliger, A.; Lu, D.; Kley, C.S.; Bischak, C.G.; Kong, Q.; Lei, T.; Dou, L.; Ginsberg, N.S.; Limmer, D.T.; et al. Intrinsic anion diffusivity in lead halide perovskites is facilitated by a soft lattice. Proc. Natl. Acad. Sci. USA 2018, 115, 11929-11934. [CrossRef]

30. Yuan, Y.; Huang, J. Ion Migration in Organometal Trihalide Perovskite and Its Impact on Photovoltaic Efficiency and Stability. Acc. Chem. Res. 2016, 49, 286-293. [CrossRef]

31. Hoke, E.T.; Slotcavage, D.J.; Dohner, E.R.; Bowring, A.R.; Karunadasa, H.I.; McGehee, M.D. Reversible photo-induced trap formation in mixed-halide hybrid perovskites for photovoltaics. Chem. Sci. 2015, 6, 613-617. [CrossRef] [PubMed]

32. Keshavarz, M.; Ottesen, M.; Wiedmann, S.; Wharmby, M.; Kuchler, R.; Yuan, H.; Debroye, E.; Steele, J.A.; Martens, J.; Hussey, N.E.; et al. Tracking Structural Phase Transitions in Lead-Halide Perovskites by Means of Thermal Expansion. Adv. Mater. 2019, 31, e1900521. [CrossRef]

33. Berhe, T.A.; Su, W.N.; Chen, C.H.; Pan, C.J.; Cheng, J.H.; Chen, H.M.; Tsai, M.C.; Chen, L.Y.; Dubale, A.A.; Hwang, B.J. Organometal halide perovskite solar cells: Degradation and stability. Energy Environ. Sci. 2016, 9, 323-356. [CrossRef]

34. Wei, J.; Wang, Q.; Huo, J.; Gao, F.; Gan, Z.; Zhao, Q.; Li, H. Mechanisms and Suppression of Photoinduced Degradation in Perovskite Solar Cells. Adv. Energy Mater. 2020, 11, 2002326. [CrossRef]

35. Zhou, Y.; Poli, I.; Meggiolaro, D.; De Angelis, F.; Petrozza, A. Defect activity in metal halide perovskites with wide and narrow bandgap. Nat. Rev. Mater. 2021, 6, 986-1002. [CrossRef]

36. Brennan, M.C.; Draguta, S.; Kamat, P.V.; Kuno, M. Light-Induced Anion Phase Segregation in Mixed Halide Perovskites. ACS Energy Lett. 2018, 3, 204-213. [CrossRef]

37. Gualdrón-Reyes, A.F.; Yoon, S.J.; Mora-Seró, I. Recent insights for achieving mixed halide perovskites without halide segregation. Curr. Opin. Electrochem. 2018, 11, 84-90. [CrossRef] 
38. Brennan, M.C.; Ruth, A.; Kamat, P.V.; Kuno, M. Photoinduced Anion Segregation in Mixed Halide Perovskites. Trends Chem. 2020, 2, 282-301. [CrossRef]

39. Knight, A.J.; Herz, L.M. Preventing phase segregation in mixed-halide perovskites: A perspective. Energy Environ. Sci. 2020, 13, 2024-2046. [CrossRef]

40. Guo, Y.; Yin, X.; Que, W.; Liu, W.; Liu, H. Toward mixed-halide perovskites: Insight into photo-induced anion phase segregation. J. Mater. Chem. C 2020, 8, 14626-14644. [CrossRef]

41. Wang, Y.; Quintana, X.; Kim, J.; Guan, X.; Hu, L.; Lin, C.-H.; Jones, B.T.; Chen, W.; Wen, X.; Gao, H.; et al. Phase segregation in inorganic mixed-halide perovskites: From phenomena to mechanisms. Photonics Res. 2020, 8, 402411. [CrossRef]

42. Kuno, M.; Brennan, M.C. What Exactly Causes Light-Induced Halide Segregation in Mixed-Halide Perovskites? Matter 2020, 2, 21-23. [CrossRef]

43. Choe, H.; Jeon, D.; Lee, S.J.; Cho, J. Mixed or Segregated: Toward Efficient and Stable Mixed Halide Perovskite-Based Devices ACS Omega 2021, 6, 24304-24315. [CrossRef] [PubMed]

44. Kamat, P.V.; Kuno, M. Halide Ion Migration in Perovskite Nanocrystals and Nanostructures. Acc. Chem. Res. 2021, 54, 520-531. [CrossRef] [PubMed]

45. Kerner, R.A.; Xu, Z.; Larson, B.W.; Rand, B.P. The role of halide oxidation in perovskite halide phase separation. Joule 2021, 5 , 2273-2295. [CrossRef]

46. Jošt, M.; Kegelmann, L.; Korte, L.; Albrecht, S. Monolithic Perovskite Tandem Solar Cells: A Review of the Present Status and Advanced Characterization Methods Toward 30\% Efficiency. Adv. Energy Mater. 2020, 10, 1904102. [CrossRef]

47. deQuilettes, D.W.; Vorpahl, S.M.; Stranks, S.D.; Nagaoka, H.; Eperon, G.E.; Ziffer, M.E.; Snaith, H.J.; Ginger, D.S. Impact of microstructure on local carrier lifetime in perovskite solar cells. Science 2015, 348, 683-686. [CrossRef]

48. deQuilettes, D.W.; Zhang, W.; Burlakov, V.M.; Graham, D.J.; Leijtens, T.; Osherov, A.; Bulovic, V.; Snaith, H.J.; Ginger, D.S.; Stranks, S.D. Photo-induced halide redistribution in organic-inorganic perovskite films. Nat. Commun. 2016, 7, 11683. [CrossRef]

49. deQuilettes, D.W.; Jariwala, S.; Burke, S.; Ziffer, M.E.; Wang, J.T.; Snaith, H.J.; Ginger, D.S. Tracking Photoexcited Carriers in Hybrid Perovskite Semiconductors: Trap-Dominated Spatial Heterogeneity and Diffusion. ACS Nano 2017, 11, 11488-11496. [CrossRef]

50. Yang, M.; Zeng, Y.; Li, Z.; Kim, D.H.; Jiang, C.S.; van de Lagemaat, J.; Zhu, K. Do grain boundaries dominate non-radiative recombination in $\mathrm{CH}_{3} \mathrm{NH}_{3} \mathrm{PbI}_{3}$ perovskite thin films? Phys. Chem. Chem. Phys. 2017, 19, 5043-5050. [CrossRef]

51. Li, C.; Guerrero, A.; Zhong, Y.; Graser, A.; Luna, C.A.M.; Kohler, J.; Bisquert, J.; Hildner, R.; Huettner, S. Real-Time Observation of Iodide Ion Migration in Methylammonium Lead Halide Perovskites. Small 2017, 13, 1701711. [CrossRef] [PubMed]

52. Li, C.; Guerrero, A.; Huettner, S.; Bisquert, J. Unravelling the role of vacancies in lead halide perovskite through electrical switching of photoluminescence. Nat. Commun. 2018, 9, 5113. [CrossRef] [PubMed]

53. Frohna, K.; Anaya, M.; Macpherson, S.; Sung, J.; Doherty, T.A.S.; Chiang, Y.H.; Winchester, A.J.; Orr, K.W.P.; Parker, J.E.; Quinn, P.D.; et al. Nanoscale chemical heterogeneity dominates the optoelectronic response of alloyed perovskite solar cells. Nat. Nanotechnol. 2021, 17, 190-196. [CrossRef]

54. Kosar, S.; Winchester, A.J.; Doherty, T.A.S.; Macpherson, S.; Petoukhoff, C.E.; Frohna, K.; Anaya, M.; Chan, N.S.; Madéo, J.; Man, M.K.L.; et al. Unraveling the varied nature and roles of defects in hybrid halide perovskites with time-resolved photoemission electron microscopy. Energy Environ. Sci. 2021, 14, 6320-6328. [CrossRef] [PubMed]

55. Jariwala, S.; Sun, H.; Adhyaksa, G.W.P.; Lof, A.; Muscarella, L.A.; Ehrler, B.; Garnett, E.C.; Ginger, D.S. Local Crystal Misorientation Influences Non-radiative Recombination in Halide Perovskites. Joule 2019, 3, 3048-3060. [CrossRef]

56. Tang, X.; van den Berg, M.; Gu, E.; Horneber, A.; Matt, G.J.; Osvet, A.; Meixner, A.J.; Zhang, D.; Brabec, C.J. Local Observation of Phase Segregation in Mixed-Halide Perovskite. Nano Lett. 2018, 18, 2172-2178. [CrossRef]

57. Zhao, Y.; Miao, P.; Elia, J.; Hu, H.; Wang, X.; Heumueller, T.; Hou, Y.; Matt, G.J.; Osvet, A.; Chen, Y.T.; et al. Strain-activated light-induced halide segregation in mixed-halide perovskite solids. Nat. Commun. 2020, 11, 6328. [CrossRef]

58. Yin, X.; Guo, Y.; Liu, J.; Que, W.; Ma, F.; Xu, K. Photoinduced Phase Segregation Leading to Evident Open-Circuit Voltage Loss in Efficient Inorganic CsPbIBr 2 Solar Cells. J. Phys. Chem. Lett. 2020, 11, 7035-7041. [CrossRef]

59. Tiede, D.O.; Calvo, M.E.; Galisteo-Lopez, J.F.; Miguez, H. Local Rearrangement of the Iodide Defect Structure Determines the Phase Segregation Effect in Mixed-Halide Perovskites. J. Phys. Chem. Lett. 2020, 11, 4911-4916. [CrossRef]

60. Yoon, S.J.; Kuno, M.; Kamat, P.V. Shift Happens. How Halide Ion Defects Influence Photoinduced Segregation in Mixed Halide Perovskites. ACS Energy Lett. 2017, 2, 1507-1514. [CrossRef]

61. Barker, A.J.; Sadhanala, A.; Deschler, F.; Gandini, M.; Senanayak, S.P.; Pearce, P.M.; Mosconi, E.; Pearson, A.J.; Wu, Y.; Srimath Kandada, A.R.; et al. Defect-Assisted Photoinduced Halide Segregation in Mixed-Halide Perovskite Thin Films. ACS Energy Lett. 2017, 2, 1416-1424. [CrossRef]

62. Belisle, R.A.; Bush, K.A.; Bertoluzzi, L.; Gold-Parker, A.; Toney, M.F.; McGehee, M.D. Impact of Surfaces on Photoinduced Halide Segregation in Mixed-Halide Perovskites. ACS Energy Lett. 2018, 3, 2694-2700. [CrossRef]

63. Knight, A.J.; Wright, A.D.; Patel, J.B.; McMeekin, D.P.; Snaith, H.J.; Johnston, M.B.; Herz, L.M. Electronic Traps and Phase Segregation in Lead Mixed-Halide Perovskite. ACS Energy Lett. 2019, 4, 75-84. [CrossRef]

64. Knight, A.J.; Patel, J.B.; Snaith, H.J.; Johnston, M.B.; Herz, L.M. Trap States, Electric Fields, and Phase Segregation in Mixed-Halide Perovskite Photovoltaic Devices. Adv. Energy Mater. 2020, 10, 1903488. [CrossRef] 
65. Motti, S.G.; Meggiolaro, D.; Barker, A.J.; Mosconi, E.; Perini, C.A.R.; Ball, J.M.; Gandini, M.; Kim, M.; De Angelis, F.; Petrozza, A. Controlling competing photochemical reactions stabilizes perovskite solar cells. Nat. Photonics 2019, 13, 532-539. [CrossRef]

66. Ruth, A.; Brennan, M.C.; Draguta, S.; Morozov, Y.V.; Zhukovskyi, M.; Janko, B.; Zapol, P.; Kuno, M. Vacancy-Mediated Anion Photosegregation Kinetics in Mixed Halide Hybrid Perovskites: Coupled Kinetic Monte Carlo and Optical Measurements. ACS Energy Lett. 2018, 3, 2321-2328. [CrossRef]

67. Datta, K.; van Gorkom, B.T.; Chen, Z.; Dyson, M.J.; van der Pol, T.P.A.; Meskers, S.C.J.; Tao, S.; Bobbert, P.A.; Wienk, M.M.; Janssen, R.A.J. Effect of Light-Induced Halide Segregation on the Performance of Mixed-Halide Perovskite Solar Cells. ACS Appl. Energy Mater. 2021, 4, 6650-6658. [CrossRef]

68. Doherty, T.A.S.; Winchester, A.J.; Macpherson, S.; Johnstone, D.N.; Pareek, V.; Tennyson, E.M.; Kosar, S.; Kosasih, F.U.; Anaya, M.; Abdi-Jalebi, M.; et al. Performance-limiting nanoscale trap clusters at grain junctions in halide perovskites. Nature 2020, 580, 360-366. [CrossRef]

69. Park, J.-S.; Calbo, J.; Jung, Y.-K.; Whalley, L.D.; Walsh, A. Accumulation of Deep Traps at Grain Boundaries in Halide Perovskites ACS Energy Lett. 2019, 4, 1321-1327. [CrossRef]

70. Tennyson, E.M.; Doherty, T.A.S.; Stranks, S.D. Heterogeneity at multiple length scales in halide perovskite semiconductors. Nat. Rev. Mater. 2019, 4, 573-587. [CrossRef]

71. Duong, T.; Mulmudi, H.K.; Wu, Y.; Fu, X.; Shen, H.; Peng, J.; Wu, N.; Nguyen, H.T.; Macdonald, D.; Lockrey, M.; et al. Light and Electrically Induced Phase Segregation and Its Impact on the Stability of Quadruple Cation High Bandgap Perovskite Solar Cells. ACS Appl. Mater. Interfaces 2017, 9, 26859-26866. [CrossRef] [PubMed]

72. Suchan, K.; Merdasa, A.; Rehermann, C.; Unger, E.L.; Scheblykin, I.G. Complex evolution of photoluminescence during phase segregation of $\mathrm{MAPb}\left(\mathrm{I}_{1-\mathrm{x}} \mathrm{Br}_{\mathrm{x}}\right)_{3}$ mixed halide perovskite. J. Lumin. 2020, 221, 117073. [CrossRef]

73. Zhong, Y.; Luna, C.A.M.; Hildner, R.; Li, C.; Huettner, S. In situ investigation of light soaking in organolead halide perovskite films. APL Mater. 2019, 7, 041114. [CrossRef]

74. Vicente, J.R.; Chen, J. Phase Segregation and Photothermal Remixing of Mixed-Halide Lead Perovskites. J. Phys. Chem. Lett. 2020, 11, 1802-1807. [CrossRef] [PubMed]

75. Bischak, C.G.; Wong, A.B.; Lin, E.; Limmer, D.T.; Yang, P.; Ginsberg, N.S. Tunable Polaron Distortions Control the Extent of Halide Demixing in Lead Halide Perovskites. J. Phys. Chem. Lett. 2018, 9, 3998-4005. [CrossRef]

76. Mao, W.; Hall, C.R.; Chesman, A.S.R.; Forsyth, C.; Cheng, Y.B.; Duffy, N.W.; Smith, T.A.; Bach, U. Visualizing Phase Segregation in Mixed-Halide Perovskite Single Crystals. Angew. Chem. Int. Ed. 2019, 58, 2893-2898. [CrossRef]

77. Bischak, C.G.; Hetherington, C.L.; Wu, H.; Aloni, S.; Ogletree, D.F.; Limmer, D.T.; Ginsberg, N.S. Origin of Reversible Photoinduced Phase Separation in Hybrid Perovskites. Nano Lett. 2017, 17, 1028-1033. [CrossRef]

78. Zhou, W.; Zhao, Y.; Zhou, X.; Fu, R.; Li, Q.; Zhao, Y.; Liu, K.; Yu, D.; Zhao, Q. Light-Independent Ionic Transport in Inorganic Perovskite and Ultrastable Cs-Based Perovskite Solar Cells. J. Phys. Chem. Lett. 2017, 8, 4122-4128. [CrossRef]

79. Rehman, W.; McMeekin, D.P.; Patel, J.B.; Milot, R.L.; Johnston, M.B.; Snaith, H.J.; Herz, L.M. Photovoltaic mixed-cation lead mixed-halide perovskites: Links between crystallinity, photo-stability and electronic properties. Energy Environ. Sci. 2017, 10, 361-369. [CrossRef]

80. Huang, J.; Xu, P.; Liu, J.; You, X.Z. Sequential Introduction of Cations Deriving Large-Grain $\mathrm{Cs}_{\mathrm{x}} \mathrm{FA}_{1-\mathrm{x}} \mathrm{PbI}_{3} \mathrm{Thin}_{\mathrm{Film}}$ for Planar Hybrid Solar Cells: Insight into Phase-Segregation and Thermal-Healing Behavior. Small 2017, 13, 1603225. [CrossRef]

81. Dang, H.X.; Wang, K.; Ghasemi, M.; Tang, M.-C.; De Bastiani, M.; Aydin, E.; Dauzon, E.; Barrit, D.; Peng, J.; Smilgies, D.-M.; et al. Multi-cation Synergy Suppresses Phase Segregation in Mixed-Halide Perovskites. Joule 2019, 3, 1746-1764. [CrossRef]

82. Beal, R.E.; Hagström, N.Z.; Barrier, J.; Gold-Parker, A.; Prasanna, R.; Bush, K.A.; Passarello, D.; Schelhas, L.T.; Brüning, K.; Tassone, C.J.; et al. Structural Origins of Light-Induced Phase Segregation in Organic-Inorganic Halide Perovskite Photovoltaic Materials. Matter 2020, 2, 207-219. [CrossRef]

83. Mathew, P.S.; Samu, G.F.; Janáky, C.; Kamat, P.V. Iodine (I) Expulsion at Photoirradiated Mixed Halide Perovskite Interface. Should I Stay or Should I Go? ACS Energy Lett. 2020, 5, 1872-1880. [CrossRef]

84. Zhang, Q.; Su, R.; Liu, X.; Xing, J.; Sum, T.C.; Xiong, Q. High-Quality Whispering-Gallery-Mode Lasing from Cesium Lead Halide Perovskite Nanoplatelets. Adv. Funct. Mater. 2016, 26, 6238-6245. [CrossRef]

85. Dar, M.I.; Jacopin, G.; Hezam, M.; Arora, N.; Zakeeruddin, S.M.; Deveaud, B.; Nazeeruddin, M.K.; Grätzel, M. Asymmetric Cathodoluminescence Emission in $\mathrm{CH}_{3} \mathrm{NH}_{3} \mathrm{PbI}_{3-\mathrm{x}} \mathrm{Br}_{\mathrm{x}}$ Perovskite Single Crystals. ACS Photonics 2016, 3, 947-952. [CrossRef]

86. Chen, W.; Mao, W.; Bach, U.; Jia, B.; Wen, X. Tracking Dynamic Phase Segregation in Mixed-Halide Perovskite Single Crystals under Two-Photon Scanning Laser Illumination. Small Methods 2019, 3, 1900273. [CrossRef]

87. Stavrakas, C.; Zhumekenov, A.A.; Brenes, R.; Abdi-Jalebi, M.; Bulovic, V.; Bakr, O.M.; Barnard, E.S.; Stranks, S.D. Probing buried recombination pathways in perovskite structures using 3D photoluminescence tomography. Energy Environ. Sci. 2018, 11, 2846-2852. [CrossRef]

88. Bischak, C.G.; Sanehira, E.M.; Precht, J.T.; Luther, J.M.; Ginsberg, N.S. Heterogeneous Charge Carrier Dynamics in OrganicInorganic Hybrid Materials: Nanoscale Lateral and Depth-Dependent Variation of Recombination Rates in Methylammonium Lead Halide Perovskite Thin Films. Nano Lett. 2015, 15, 4799-4807. [CrossRef]

89. Xiao, C.; Li, Z.; Guthrey, H.; Moseley, J.; Yang, Y.; Wozny, S.; Moutinho, H.; To, B.; Berry, J.J.; Gorman, B.; et al. Mechanisms of Electron-Beam-Induced Damage in Perovskite Thin Films Revealed by Cathodoluminescence Spectroscopy. J. Phys. Chem. C 2015, 119, 26904-26911. [CrossRef] 
90. Stranks, S.D. Multimodal microscopy characterization of halide perovskite semiconductors: Revealing a new world (dis)order. Matter 2021, 4, 3852-3866. [CrossRef]

91. Li, W.; Rothmann, M.U.; Liu, A.; Wang, Z.; Zhang, Y.; Pascoe, A.R.; Lu, J.; Jiang, L.; Chen, Y.; Huang, F.; et al. Phase Segregation Enhanced Ion Movement in Efficient Inorganic CsPbIBr 2 Solar Cells. Adv. Energy Mater. 2017, 7, 1700946. [CrossRef]

92. Kumar, V.; Nisika; Kumar, M. Temporal-spatial-energy resolved advance multidimensional techniques to probe photovoltaic materials from atomistic viewpoint for next-generation energy solutions. Energy Environ. Sci. 2021, 14, 4760-4802. [CrossRef]

93. Hidalgo, J.; Castro-Méndez, A.F.; Correa-Baena, J.P. Imaging and Mapping Characterization Tools for Perovskite Solar Cells. Adv. Energy Mater. 2019, 9, 1900444. [CrossRef]

94. Funk, H.; Shargaieva, O.; Eljarrat, A.; Unger, E.L.; Koch, C.T.; Abou-Ras, D. In Situ TEM Monitoring of Phase-Segregation in Inorganic Mixed Halide Perovskite. J. Phys. Chem. Lett. 2020, 11, 4945-4950. [CrossRef]

95. Notthoff, C.; Winterer, M.; Beckel, A.; Geller, M.; Heindl, J. Spatial high resolution energy dispersive X-ray spectroscopy on thin lamellas. Ultramicroscopy 2013, 129, 30-35. [CrossRef]

96. Hentz, O.; Zhao, Z.; Gradecak, S. Impacts of Ion Segregation on Local Optical Properties in Mixed Halide Perovskite Films. Nano Lett. 2016, 16, 1485-1490. [CrossRef]

97. Frolova, L.A.; Luchkin, S.Y.; Lekina, Y.; Gutsev, L.G.; Tsarev, S.A.; Zhidkov, I.S.; Kurmaev, E.Z.; Shen, Z.X.; Stevenson, K.J.; Aldoshin, S.M.; et al. Reversible $\mathrm{Pb}^{2+} / \mathrm{Pb}^{0}$ and $\mathrm{I}^{-} / \mathrm{I}^{3-}$ Redox Chemistry Drives the Light-Induced Phase Segregation in All-Inorganic Mixed Halide Perovskites. Adv. Energy Mater. 2021, 11, 2002934. [CrossRef]

98. Melitz, W.; Shen, J.; Kummel, A.C.; Lee, S. Kelvin probe force microscopy and its application. Surf. Sci. Rep. 2011, 66, 1-27. [CrossRef]

99. Gomez, A.; Sanchez, S.; Campoy-Quiles, M.; Abate, A. Topological distribution of reversible and non-reversible degradation in perovskite solar cells. Nano Energy 2018, 45, 94-100. [CrossRef]

100. Samu, G.F.; Balog, A.; De Angelis, F.; Meggiolaro, D.; Kamat, P.V.; Janaky, C. Electrochemical Hole Injection Selectively Expels Iodide from Mixed Halide Perovskite Films. J. Am. Chem. Soc. 2019, 141, 10812-10820. [CrossRef]

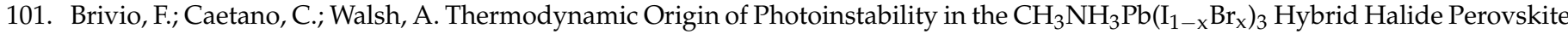
Alloy. J. Phys. Chem. Lett. 2016, 7, 1083-1087. [CrossRef] [PubMed]

102. Draguta, S.; Sharia, O.; Yoon, S.J.; Brennan, M.C.; Morozov, Y.V.; Manser, J.S.; Kamat, P.V.; Schneider, W.F.; Kuno, M. Rationalizing the light-induced phase separation of mixed halide organic-inorganic perovskites. Nat. Commun. 2017, 8, 200. [CrossRef] [PubMed]

103. Pavlovetc, I.M.; Ruth, A.; Gushchina, I.; Ngo, L.; Zhang, S.; Zhang, Z.; Kuno, M. Distinguishing Models for Mixed Halide Lead Perovskite Photosegregation via Terminal Halide Stoichiometry. ACS Energy Lett. 2021, 6, 2064-2071. [CrossRef]

104. Chen, Z.; Brocks, G.; Tao, S.; Bobbert, P.A. Unified theory for light-induced halide segregation in mixed halide perovskites. Nat. Commun. 2021, 12, 2687. [CrossRef] [PubMed]

105. Wagemaker, M.; Singh, D.P.; Borghols, W.J.; Lafont, U.; Haverkate, L.; Peterson, V.K.; Mulder, F.M. Dynamic solubility limits in nanosized olivine LiFePO. J. Am. Chem. Soc. 2011, 133, 10222-10228. [CrossRef] [PubMed]

106. Bai, P.; Cogswell, D.A.; Bazant, M.Z. Suppression of phase separation in LiFePO $_{4}$ nanoparticles during battery discharge. Nano Lett. 2011, 11, 4890-4896. [CrossRef]

107. Zhu, C.; Mu, X.; Popovic, J.; Weichert, K.; van Aken, P.A.; Yu, Y.; Maier, J. Lithium potential variations for metastable materials: Case study of nanocrystalline and amorphous $\mathrm{LiFePO}_{4}$. Nano Lett. 2014, 14, 5342-5349. [CrossRef]

108. Wang, Y.-R.; Yeong Kim, G.; Kotomin, E.; Moia, D.; Maier, J. Photo de-mixing in mixed halide perovskites: The roles of ions and electrons. J. Phys. Energy 2022. [CrossRef]

109. Wang, X.; Ling, Y.; Lian, X.; Xin, Y.; Dhungana, K.B.; Perez-Orive, F.; Knox, J.; Chen, Z.; Zhou, Y.; Beery, D.; et al. Suppressed phase separation of mixed-halide perovskites confined in endotaxial matrices. Nat. Commun. 2019, 10, 695. [CrossRef]

110. Babbe, F.; Masquelier, E.; Zheng, Z.; Sutter-Fella, C.M. Flash Formation of I-Rich Clusters during Multistage Halide Segregation Studied in MAPbI1.5Br1.5. J. Phys. Chem. C 2020, 124, 24608-24615. [CrossRef]

111. Mao, W.; Hall, C.R.; Bernardi, S.; Cheng, Y.B.; Widmer-Cooper, A.; Smith, T.A.; Bach, U. Light-induced reversal of ion segregation in mixed-halide perovskites. Nat. Mater. 2021, 20, 55-61. [CrossRef] [PubMed]

112. Guo, Y.; Yin, X.; Liu, D.; Liu, J.; Zhang, C.; Xie, H.; Yang, Y.; Que, W. Photoinduced Self-healing of Halide Segregation in Mixed-halide Perovskites. ACS Energy Lett. 2021, 6, 2502-2511. [CrossRef]

113. Cho, J.; Kamat, P.V. Photoinduced Phase Segregation in Mixed Halide Perovskites: Thermodynamic and Kinetic Aspects of Cl-Br Segregation. Adv. Opt. Mater. 2020, 9, 2001440. [CrossRef]

114. Elmelund, T.; Seger, B.; Kuno, M.; Kamat, P.V. How Interplay between Photo and Thermal Activation Dictates Halide Ion Segregation in Mixed Halide Perovskites. ACS Energy Lett. 2019, 5, 56-63. [CrossRef]

115. van de Goor, T.W.J.; Liu, Y.; Feldmann, S.; Bourelle, S.A.; Neumann, T.; Winkler, T.; Kelly, N.D.; Liu, C.; Jones, M.A.; Emge, S.P.; et al. Impact of Orientational Glass Formation and Local Strain on Photo-Induced Halide Segregation in Hybrid Metal-Halide Perovskites. J. Phys. Chem. C 2021, 125, 15025-15034. [CrossRef]

116. DuBose, J.T.; Kamat, P.V. TiO 2 -Assisted Halide Ion Segregation in Mixed Halide Perovskite Films. J. Am. Chem. Soc. 2020, 142, 5362-5370. [CrossRef] 
117. Al-Ashouri, A.; Kohnen, E.; Li, B.; Magomedov, A.; Hempel, H.; Caprioglio, P.; Marquez, J.A.; Morales Vilches, A.B.; Kasparavicius, E.; Smith, J.A.; et al. Monolithic perovskite/silicon tandem solar cell with $>29 \%$ efficiency by enhanced hole extraction. Science 2020, 370, 1300-1309. [CrossRef]

118. Cho, J.; DuBose, J.T.; Mathew, P.S.; Kamat, P.V. Electrochemically induced iodine migration in mixed halide perovskites: Suppression through chloride insertion. Chem. Commun. 2021, 57, 235-238. [CrossRef]

119. Shi, T.; Yin, W.-J.; Hong, F.; Zhu, K.; Yan, Y. Unipolar self-doping behavior in perovskite $\mathrm{CH}_{3} \mathrm{NH}_{3} \mathrm{PbBr}_{3}$. Appl. Phys. Lett. 2015, 106, 103902. [CrossRef]

120. Kim, G.Y.; Senocrate, A.; Yang, T.Y.; Gregori, G.; Gratzel, M.; Maier, J. Large tunable photoeffect on ion conduction in halide perovskites and implications for photodecomposition. Nat. Mater. 2018, 17, 445-449. [CrossRef]

121. Beal, R.E.; Slotcavage, D.J.; Leijtens, T.; Bowring, A.R.; Belisle, R.A.; Nguyen, W.H.; Burkhard, G.F.; Hoke, E.T.; McGehee, M.D. Cesium Lead Halide Perovskites with Improved Stability for Tandem Solar Cells. J. Phys. Chem. Lett. 2016, 7, 746-751. [CrossRef]

122. Xu, J.; Boyd, C.C.; Yu, Z.J.; Palmstrom, A.F.; Witter, D.J.; Larson, B.W.; France, R.M.; Werner, J.; Harvey, S.P.; Wolf, E.J.; et al Triple-halide wide-band gap perovskites with suppressed phase segregation for efficient tandems. Science 2020, 367, 1097-1104. [CrossRef]

123. Knight, A.J.; Borchert, J.; Oliver, R.D.J.; Patel, J.B.; Radaelli, P.G.; Snaith, H.J.; Johnston, M.B.; Herz, L.M. Halide Segregation in Mixed-Halide Perovskites: Influence of A-Site Cations. ACS Energy Lett. 2021, 6, 799-808. [CrossRef]

124. Elmelund, T.; Scheidt, R.A.; Seger, B.; Kamat, P.V. Bidirectional Halide Ion Exchange in Paired Lead Halide Perovskite Films with Thermal Activation. ACS Energy Lett. 2019, 4, 1961-1969. [CrossRef] 